\title{
Periodic solutions in unbounded domains for the Boussinesq
}

\author{
system *
}

Elder Jesús VILLAMIZAR-ROA

Corresponding author, Escuela de Matemáticas, Universidad Nacional de Colombia, Sede Medellín A.A. 3840, Medellín, Colombia, ejvillamizarr@unalmed.edu.co

María Ángeles RODRÍGUEZ-BELLIDO

Dpto. Ecuaciones Diferenciales y Análisis Numérico, Universidad de Sevilla, Aptdo. 1160 -41080 Sevilla, Spain, angeles@us.es

$\&$

Marko Antonio ROJAS-MEDAR

Dpto. de Ciencias Básicas, Facultad de Ciencias, Universidad del Bío Bío, Campus Fernando May, Casilla 447, Chillán, Chile, marko@ueubiobio.cl

\begin{abstract}
Assuming that the external forces of the system are small enough, the reference temperature being a periodic function, we study the existence, the uniqueness and the regularity of time-periodic solutions for the Boussinesq equations in several classes of unbounded domains of $\mathbb{R}^{n}$. Our analysis is based on the framework of weak- $L^{p}$ spaces.
\end{abstract}

Keywords: Boussinesq equations, Strong Periodic Solutions, Unbounded domains. MRSubClass: 35B35, 35B40, 35Q35, 76D03.

\section{Introduction}

Let $\Omega$ be as either the whole space $\mathbb{R}^{n}, n \geq 3$, either the half space $\mathbb{R}_{+}^{n}, n \geq 3$, either a bounded domain in $\mathbb{R}^{n}, n \geq 3$, or an exterior domain in $\mathbb{R}^{n}, n \geq 4$, with boundary $\partial \Omega$ of class $C^{2+\mu}(\mu>0)$.

${ }^{*}$ The second and third authors have been partially supported by M.E.C. (Spain), Project MTM2006-07932. The second author has been partially supported by by Junta de Andalucía, Project P06-FQM-02373. The third author has been partially supported by Fondecyt-Chile, No. 1080628. 
We consider the following nonstationary Boussinesq equations in $\Omega$ :

$$
\begin{aligned}
\frac{\partial \mathbf{u}}{\partial t}-\nu \Delta \mathbf{u}+(\mathbf{u} \cdot \nabla) \mathbf{u}+\frac{1}{\rho} \nabla p & =\beta \theta \mathbf{g}+\mathbf{\Psi}, \quad \mathbf{x} \in \Omega, t \in \mathbb{R}, \\
\nabla \cdot \mathbf{u} & =0, \quad \mathbf{x} \in \Omega, t \in \mathbb{R}, \\
\frac{\partial \theta}{\partial t}-\chi \Delta \theta+(\mathbf{u} \cdot \nabla) \theta & =f, \quad \mathbf{x} \in \Omega, t \in \mathbb{R} \\
\mathbf{u} & =\mathbf{0} \text { on } \partial \Omega \\
\theta & =0 \text { on } \partial \Omega,
\end{aligned}
$$

where $\mathbf{g}$ represents the gravitational field at $\mathbf{x}, f$ is the reference temperature, $\boldsymbol{\Psi}$ is an external force and $\rho, \nu, \beta, \chi$ are positive physical constants which denote, respectively, the density, the kinematic viscosity, the coefficient of volume expansion and the thermal conductance. The unknowns are $\mathbf{u}(\mathbf{x}, t) \in \mathbb{R}^{n}, p(\mathbf{x}, t) \in \mathbb{R}$ and $\theta(\mathbf{x}, t) \in \mathbb{R}$ representing respectively, the velocity field, the pressure and the temperature of the fluid at point $(x, t) \in \Omega \times \mathbb{R}$. Boussinesq equations describe the evolution of the temperature and velocity field of a viscous incompressible Newtonian fluid. For an extensive discussion on the physical origin of the equations (1.1)-(1.3), see [4].

We are interested in the study of the time-periodic solutions for the system (1.1)-(1.5) when the reference temperature is a periodic function with the same period. Without loss of generality, we have taken the constants $\rho, \nu, \beta, \chi$ equal to one. To avoid some technical complexities in the study of (1.1)-(1.5), throughout this paper we assume $\boldsymbol{\Psi}=\mathbf{0}$. Several works have been made in the mathematical analysis of system (1.1)-(1.5); see, for instance, [3], [5], [7], [13], [6] and papers cited therein. The time-periodic solutions for the Boussinesq equations in bounded domains was considered in [13]. The analysis was made via the Galerkin's method. Indeed, in [13] it was considered a class of nonlinear evolution equations in a separable Hilbert space generalizing several models of hydrodynamics. However, the study of periodic solutions for system (1.1)-(1.5) has not been investigated in unbounded domains. Hence, the purpose of the present paper is to prove the existence and uniqueness of strong periodic solutions for problem (1.1)-(1.5) in the framework of Semigroups Theory on the Lorentz spaces, more explicitly, on the theory of weak-L $\mathbf{L}^{p}$ spaces. We construct the time-periodic solutions using $\mathbf{L}^{p, q}-\mathbf{L}^{r, s}$ estimates for the semigroups generated by the Stokes and Laplace operators. If $\Omega$ is an exterior domain, we need to assume $n \geq 4$ in order to obtain the gradient bounds for the semigroups generated by the Stokes and the Laplace operators in $\mathbf{L}^{(p, \infty)}$ (see Lemma 3.2).

This work is motivated by the existence results of periodic solutions for the Navier-Stokes equations. In unbounded domains, this subject has been investigated in [11], [14], [15], [18] and [19]. In particular, in [14] was proved the existence of a unique time-periodic solution on the whole space $\mathbb{R}^{3}$ for small external force. The problem in the half-space $\mathbb{R}_{+}^{3}$ was considered in [15]. In [11], making use of $\mathbf{L}^{p}-\mathbf{L}^{r}$ estimates for the semigroup generated by the Stokes operator, time-periodic solutions were constructed for small time-periodic forces. The stability 
of these solutions was considered in [18]. However, the existence of strong time-periodic solutions in general unbounded domains is still an open problem. More complete references, including results for bounded domains, can be found in [11], [14], [15].

This paper is organized as follows. In Section $§ 2$, we give some preliminaries about Lorentz spaces and state our main results. Section $\S 3$ is devoted to prove the existence and the uniqueness of strong periodic solutions.

\section{Preliminaries and Results}

Before stating our results we introduce some functional spaces. $\mathbf{C}_{0, \sigma}^{\infty}(\Omega)$ denotes the set of all $C^{\infty}$-real functions $\varphi=\left(\varphi_{1}, \ldots, \varphi_{n}\right)$ with compact support in $\Omega$, such that $\operatorname{div} \varphi=0$. The closure of $\mathbf{C}_{0, \sigma}^{\infty}$ with respect to norm $\mathbf{L}^{r}, 1<r<\infty$, is denoted by $\mathbf{L}_{\sigma}^{r}(\Omega)$. Let us recall the Helmholtz decomposition: $\mathbf{L}^{r}(\Omega)=\mathbf{L}_{\sigma}^{r}(\Omega) \oplus \mathbf{G}^{r}(\Omega), 1<r<\infty$, where $\mathbf{G}^{r}(\Omega)=\left\{\nabla p \in \mathbf{L}^{r}(\Omega)\right.$ : $\left.p \in L_{l o c}^{r}(\bar{\Omega})\right\}$ (c.f. [8]). $P_{r}$ denotes the projection operator from $\mathbf{L}^{r}(\Omega)$ onto $\mathbf{L}_{\sigma}^{r}(\Omega)$. The Stokes operator $\mathbf{A}_{r}=-P_{r} \Delta$ with domain $D\left(\mathbf{A}_{r}\right)=\left\{\mathbf{u} \in \mathbf{H}^{2, r}(\Omega):\left.\mathbf{u}\right|_{\partial \Omega}=\mathbf{0}\right\} \cap \mathbf{L}_{\sigma}^{r}$. It is well known that $-\mathbf{A}_{r}$ generates a uniformly bounded analytic semigroup $\left\{e^{-t \mathbf{A}_{r}}\right\}_{t \geq 0}$ of class $C_{0}$ in $\mathbf{L}_{\sigma}^{r}$ (c.f. $[9])$.

We denote by $B_{q}$ the Laplace operator in $L^{q}(\Omega), 1<q<\infty$, with homogeneous Dirichlet boundary conditions: $B_{q}=-\Delta$ with domain $D\left(B_{q}\right)=W^{2, q}(\Omega) \cap W_{0}^{1, q}(\Omega)$. We recall that the operator $-B_{q}$ generates a uniformly bounded analytic semigroup $\left\{e^{-t B_{q}}\right\}_{t \geq 0}$ in $L^{q}(\Omega)$ of class $C_{0}$.

Now we introduce some preliminaries about the Lorentz spaces. For details see [1]. Let $1<p \leq \infty$ and $1 \leq q \leq \infty$. A Lebesgue measurable function $f$ defined on a domain $\Omega \subset \mathbb{R}^{n}$ belongs to Lorentz space $L^{(p, q)}(\Omega)$ if the quantity

$$
\|f\|_{(p, q)}=\left\{\begin{array}{lll}
\left(\frac{q}{p} \int_{0}^{\infty}\left[t^{\frac{1}{p}} f^{* *}(t)\right]^{q} \frac{d t}{t}\right)^{\frac{1}{q}} & , \text { if } & 1<p<\infty, 1 \leq q<\infty \\
\sup _{t>0} t^{\frac{1}{p}} f^{* *}(t) & , \text { if } \quad 1<p \leq \infty, q=\infty
\end{array},\right.
$$

is finite, where

$$
f^{* *}(t)=\frac{1}{t} \int_{0}^{t} f^{*}(s) d s, \quad f^{*}(t)=\inf \{s>0: m\{\mathbf{x} \in \Omega:|f(\mathbf{x})|>s\} \leq t\}, t>0,
$$

with $m$ denoting the Lebesgue measure on $\mathbb{R}^{n}$. The spaces $L^{(p, q)}$ with the norm $\|f\|_{(p, q)}$ are Banach spaces. Note that $L^{p}(\Omega)=L^{(p, p)}(\Omega)$. When $q=\infty, L^{(p, \infty)}(\Omega)$ are called the Marcinkiewicz spaces or weak- $L^{p}$ spaces. Moreover, $L^{\left(p, q_{1}\right)}(\Omega) \subset L^{p}(\Omega) \subset L^{\left(p, q_{2}\right)}(\Omega) \subset L^{(p, \infty)}(\Omega)$ for $0<$ $q_{1} \leq p \leq q_{2} \leq \infty$. We recall that the space $C_{0}^{\infty}(\Omega)$ is not dense in $L^{(p, \infty)}(\Omega)$. Borchers and Miyakawa [2] established the following Helmholtz decomposition of the Lorentz spaces extending the operator $P_{r}$ to a bounded operator on $\mathbf{L}^{(r, d)}(\Omega)$, which we denote by $P_{r, d}$. Setting $\mathbf{L}_{\sigma}^{(r, d)}(\Omega)=\operatorname{Range}\left(P_{r, d}\right)$ and $\mathbf{G}^{(r, d)}(\Omega)=\operatorname{Kernel}\left(P_{r, d}\right)$, then $\mathbf{L}^{(r, d)}(\Omega)=\mathbf{L}_{\sigma}^{(r, d)}(\Omega) \oplus \mathbf{G}^{(r, d)}(\Omega)$, 
with $\mathbf{L}_{\sigma}^{(r, d)}(\Omega)=\left\{\mathbf{u} \in \mathbf{L}^{(r, d)}(\Omega): \nabla \cdot \mathbf{u}=0,\left.\mathbf{u} \cdot \mathbf{n}\right|_{\partial \Omega}=0\right\}$ and $\mathbf{G}^{(r, d)}(\Omega)=\left\{\nabla v \in \mathbf{L}^{(r, d)}(\Omega)\right.$ : $\left.v \in L_{l o c}^{(r, d)}(\bar{\Omega})\right\}$. For simplicity, we shall abbreviate the projection operator and the Stokes and Laplace operators on Lorentz spaces by $P, \mathbf{A}, B$, respectively. In view of [2], the operators $-\mathbf{A},-B$ generate bounded analytic semigroups on $\mathbf{L}_{\sigma}^{(p, q)}(\Omega)$ and $L^{(p, q)}(\Omega)$, respectively. However, we recall that if $q=\infty$, this semigroups are not strongly continuous at $t=0$.

Applying the operator $P$ on the equations (1.1)-(1.2), from (1.1)-(1.5) we obtain the following problem of parabolic type:

$$
\begin{aligned}
\mathbf{u}_{t}+\mathbf{A} \mathbf{u}+P[(\mathbf{u} \cdot \nabla) \mathbf{u}] & =P(\theta \mathbf{g}), \quad t \in \mathbb{R} \\
\theta_{t}+B \theta+(\mathbf{u} \cdot \nabla) \theta & =f, \quad t \in \mathbb{R} .
\end{aligned}
$$

The system (2.6)-(2.7), with periodic in time conditions, has associated the following system of integral equations

$$
\begin{aligned}
\mathbf{u}(t) & =-\int_{-\infty}^{t} e^{-(t-s) \mathbf{A}} P[(\mathbf{u} \cdot \nabla) \mathbf{u}] d s+\int_{-\infty}^{t} e^{-(t-s) \mathbf{A}} P(\theta \mathbf{g}) d s \\
\theta(t) & =-\int_{-\infty}^{t} e^{-(t-s) B}(\mathbf{u} \cdot \nabla) \theta d s+\int_{-\infty}^{t} e^{-(t-s) B} f d s .
\end{aligned}
$$

Throughout this paper we assume the following assumptions on the external force $f$ and the field g:

\section{Assumption 1.}

(CASE 1). If $\Omega$ is either the whole space $\mathbb{R}^{n}$, a bounded domain in $\mathbb{R}^{n}$, with boundary of class $C^{2+\mu}(\mu>0)$, or the half space $\mathbb{R}_{+}^{n}, n \geq 3$, we consider $r, \tilde{r}, q, \tilde{q}$ verifying $2<r, \tilde{r}<n, \frac{n}{2}<$ $q, \tilde{q}<n, \frac{1}{r}-\frac{1}{\tilde{r}}<\min \left\{\frac{2}{n}-\frac{1}{q}, \frac{2}{n}-\frac{1}{\tilde{q}}\right\}$.

(CASE 2). If $\Omega$ is an exterior domain in $\mathbb{R}^{n}, n \geq 4$, with boundary of class $C^{2+\mu}(\mu>0)$, we consider $r, \tilde{r}, q, \tilde{q}$ such that $\frac{2 n}{(n-1)} \leq r, \tilde{r}<n, \frac{n}{2}<q, \tilde{q}<n, \quad \frac{1}{r}-\frac{1}{\tilde{r}}<\min \left\{\frac{2}{n}-\frac{1}{q}, \frac{2}{n}-\frac{1}{\tilde{q}}\right\}$.

For each $r, \tilde{r}$ and $q, \tilde{q}$ we assume that $f$ satisfies

$$
f \in B C\left(\mathbb{R} ; L^{(\tilde{p}, \infty)}(\Omega) \cap L^{(\tilde{l}, \infty)}(\Omega)\right),
$$

for $1<\tilde{p}, \tilde{l}<\infty$ with $\frac{1}{\tilde{r}}+\frac{2}{n}<\frac{1}{\tilde{p}}, \quad \frac{1}{\tilde{q}}<\frac{1}{\tilde{l}}<\frac{1}{\tilde{q}}+\frac{1}{n}$ provided $n \geq 4$ in both CASES $(1,2)$. (Note that as $n<2 \tilde{q}, \tilde{r}<n$, then the inequality $1 / \tilde{q}<1 / \tilde{l}<1 / \tilde{q}+1 / n$ implies that $1 / \tilde{l}<2 / n+1 / \tilde{r})$. If $n=3$, in the CASE 1 , we assume that $f$ satisfies

$$
\left\{\begin{array}{l}
f \in B C\left(\mathbb{R}, L^{(\tilde{l}, \infty)}(\Omega)\right) \text { such that } \\
f(s)=B_{\tilde{p}, \infty}^{\delta} h(s) \text { for some } h \in B C\left(\mathbb{R}, D\left(B_{\tilde{p}, \infty}^{\delta}\right)\right),
\end{array}\right.
$$


for $1<\tilde{p}<\min \{\tilde{r}, \tilde{q}\}$, and $\delta>0$ satisfying $\frac{3}{2 \tilde{p}}+\delta>1+\max \left\{1+\frac{3}{2 \tilde{r}}, \frac{1}{2}+\frac{3}{2 \tilde{q}}\right\}$ and $1 / \tilde{q}<1 / \tilde{l}<$ $1 / \tilde{q}+1 / 3$, where $B_{\tilde{p}, \infty}^{\delta}$ denotes the power $\delta$ of the operator $B$ on $L^{\tilde{p}, \infty}$. With respect to the field $\mathrm{g}$ we make the following assumptions:

$$
\mathbf{g} \in \mathbf{L}^{(a, \infty)}(\Omega) \cap \mathbf{L}^{(b, \infty)}(\Omega)
$$

where $a$ and $b$ are such that: $\frac{1}{a}>\frac{2}{n}+\frac{1}{r}-\frac{1}{\tilde{r}}, \quad \frac{1}{b}<\frac{1}{n}+\frac{1}{q}-\frac{1}{\tilde{r}}, \quad(b>1, a>1)$.

Remark 2.1 Condition (2.11) can be replaced by $f(s)=\nabla \cdot \boldsymbol{G}(s), \boldsymbol{G}(s)=\left(G_{1}, \ldots, G_{n}\right) \in$ $B C\left(\mathbb{R} ; \mathbf{L}^{(\tilde{p}, \infty)}(\Omega)\right)$ with $\nabla \boldsymbol{G}(t) \in B C\left(\mathbb{R} ; \mathbf{L}^{(\tilde{p}, \infty)}(\Omega)\right)^{n}$ for $1<\tilde{p}<\infty$ with $1 / \tilde{r}+1 / 3<1 / \tilde{p}$. This implies that $f(s)=\Delta h(s)$ for some $h \in B C\left(\mathbb{R} ; D\left(B_{\tilde{p}, \infty}\right)\right)$.

Our main results are stated as follows:

Theorem 2.2 Let $f$ be a periodic function with period $\tau>0$ (i.e, for all $t \in \mathbb{R}, f(t)=f(t+\tau)$ ) satisfying Assumption 1. Then, if the quantities

$$
\begin{aligned}
\sup _{s \in \mathbb{R}}\|f(s)\|_{(\tilde{p}, \infty)}+\sup _{s \in \mathbb{R}}\|f(s)\|_{(\tilde{l}, \infty)}, & n \geq 4, \text { in the CASES } 1 \text { and 2, } \\
\sup _{s \in \mathbb{R}}\|h(s)\|_{(\tilde{p}, \infty)}+\sup _{s \in \mathbb{R}}\|f(s)\|_{(\tilde{l}, \infty)}, & n=3, \text { in the CASE 1, } \\
\|\mathbf{g}\|_{(b, \infty)}+\|\mathbf{g}\|_{(a, \infty)}, & \text { in the CASES 1 and 2, }
\end{aligned}
$$

are small enough, then there exists a periodic solution $(\mathbf{u}, \theta)$ of (2.8),(2.9), with the same period $\tau$ of the external force, in the class $\mathbf{u} \in B C\left(\mathbb{R} ; \mathbf{L}_{\sigma}^{(r, \infty)}(\Omega)\right), \theta \in B C\left(\mathbb{R} ; L^{(\tilde{r}, \infty)}(\Omega)\right)$, with $\nabla \mathbf{u} \in$ $B C\left(\mathbb{R} ; \mathbf{L}^{(q, \infty)}(\Omega)\right)^{n}, \nabla \theta \in B C\left(\mathbb{R} ; L^{(\tilde{q}, \infty)}(\Omega)\right)^{n}$. Moreover, within this class, if

$$
\sup _{s \in \mathbb{R}}\|\mathbf{u}(s)\|_{(r, \infty)}+\sup _{s \in \mathbb{R}}\|\nabla \mathbf{u}(s)\|_{(q, \infty)}, \quad \sup _{s \in \mathbb{R}}\|\theta(s)\|_{(\tilde{r}, \infty)}+\sup _{s \in \mathbb{R}}\|\nabla \theta(s)\|_{(\tilde{q}, \infty)}
$$

are small enough, then the solution is unique.

Theorem 2.3 Under the assumptions of Theorem 2.2, if $f$ is Hölder continuous with values in $L^{\left(r^{*}, \infty\right)}(\Omega)$ and $\mathbf{g} \in \mathbf{L}^{\left(r^{*}, \infty\right)}(\Omega)$, then, for all $n<r^{*}<q^{*}=n q /(n-q)$, the periodic solution given by Theorem 2.2 satisfies

1. $\mathbf{u} \in B C\left(\mathbb{R} ; \mathbf{L}_{\sigma}^{(n, \infty)}(\Omega)\right) \cap C^{1}\left(\mathbb{R} ; \mathbf{L}_{\sigma}^{r^{*}}(\Omega)\right), \theta \in B C\left(\mathbb{R} ; L^{(n, \infty)}(\Omega)\right) \cap C^{1}\left(\mathbb{R} ; L^{r^{*}}(\Omega)\right)$.

2. $\mathbf{A}_{n} \mathbf{u} \in C\left(\mathbb{R} ; \mathbf{L}_{\sigma}^{r^{*}}(\Omega)\right), B_{n} \theta \in C\left(\mathbb{R} ; L^{r^{*}}(\Omega)\right)$.

3. For all $t \in \mathbb{R}$, (2.6) and (2.7) are satisfied in $\mathbf{L}_{\sigma}^{r^{*}}(\Omega)$ and $L^{r^{*}}(\Omega)$, respectively. 


\section{Existence, Uniqueness and Regularity of Periodic Solutions}

In this section we prove Theorem 2.2 and Theorem 2.3. Let us first recall the Hölder's inequality and some $L^{(r, \infty)}-L^{(p, \infty)}$ estimates for the semigroups $\left\{e^{-t \mathbf{A}}\right\}_{t \geq 0},\left\{e^{-t B}\right\}_{t \geq 0}$.

Proposition 3.1 (Generalized Hölder's inequality [16]) Let $1<p_{1}, p_{2}, r<\infty, f \in$ $L^{\left(p_{1}, q_{1}\right)}(\Omega)$ and $g \in L^{\left(p_{2}, q_{2}\right)}(\Omega)$ where $\frac{1}{p_{1}}+\frac{1}{p_{2}}<1$, then fg belongs to $L^{(r, s)}(\Omega)$ where $\frac{1}{r}=\frac{1}{p_{1}}+\frac{1}{p_{2}}$, and $s \geq 1$ is any number such that $\frac{1}{q_{1}}+\frac{1}{q_{2}} \geq \frac{1}{s}$. Moreover,

$$
\|f g\|_{(r, s)} \leq C(r)\|f\|_{\left(p_{1}, q_{1}\right)}\|g\|_{\left(p_{2}, q_{2}\right)} .
$$

Lemma 3.2 ([2], [19]).

1. Let $\Omega$ be either the whole space $\mathbb{R}^{n}$, a bounded domain in $\mathbb{R}^{3}$ with boundary $\partial \Omega$ of class $C^{2+\mu}(\mu>0)$, or the half space $\mathbb{R}_{+}^{n}, n \geq 3$. Then

$$
\left\|\nabla^{j} e^{-t A} \mathbf{a}\right\|_{(r, 1)} \leq c t^{-n / 2(1 / p-1 / r)-j / 2}\|\mathbf{a}\|_{(p, 1)}, 1<p \leq r<\infty,
$$

for all $\mathbf{a} \in \mathbf{L}_{\sigma}^{(p, 1)}(\Omega), j=0,1$ and all $t>0$, where $c=c(n, p, r)$.

2. Let $\Omega$ be an exterior domain in $\mathbb{R}^{n}, n \geq 4$ with boundary $\partial \Omega$, of class $C^{2+\mu}(\mu>0)$. Then

$$
\begin{aligned}
\left\|e^{-t A} \mathbf{a}\right\|_{(r, 1)} & \leq c t^{-n / 2(1 / p-1 / r)}\|\mathbf{a}\|_{(p, 1)}, 1<p \leq r<\infty, \\
\left\|\nabla e^{-t A} \mathbf{a}\right\|_{(r, 1)} & \leq c t^{-n / 2(1 / p-1 / r)-1 / 2}\|\mathbf{a}\|_{(p, 1)}, 1<p \leq r \leq n,
\end{aligned}
$$

for all $\mathbf{a} \in \mathbf{L}_{\sigma}^{(p, 1)}(\Omega)$ and all $t>0$, where $c=c(n, p, r)$.

Remark 3.3 Estimates in Lemma 3.2 for $e^{-t A} \mathbf{a}$ and $\nabla e^{-t A} \mathbf{a}$ in the norm $L^{(r, \infty)}$ with respect to the data in $L^{(p, \infty)}$ are true, because they are obtained by duality. Similar estimates hold for the semigroup $\left\{e^{-t B}\right\}_{t \geq 0}$. The estimates above hold in the particular case of $L^{p}$ spaces (c.f. [11]).

Lemma 3.4 ([2]). Let $\Omega$ be as the CASE 1 and CASE 2 and suppose that $1<q<n, 1 \leq d \leq \infty$ and $q^{*}=n q /(n-q)$. If $\phi \in L^{(p, \infty)}(\Omega)$ for some $p<\infty$ and $\nabla \phi \in L^{(q, d)}(\Omega)^{n}$, then $\phi \in L^{\left(q^{*}, d\right)}(\Omega)$ and the estimate $\|\phi\|_{\left(q^{*}, d\right)} \leq C\|\nabla \phi\|_{(q, d)}$ holds with $C>0$ independent of $\phi$.

We denote by $X$ the space of scalar functions $\left\{u \in B C\left(\mathbb{R} ; L^{(\tilde{r}, \infty)}\right): \nabla u \in B C\left(\mathbb{R} ; L^{(\tilde{q}, \infty)}\right)^{n}\right\}$ with the norm $\|\cdot\|_{X}$ defined as

$$
\|u\|_{X} \equiv \sup _{s \in \mathbb{R}}\|u(s)\|_{(\tilde{r}, \infty)}+\sup _{s \in \mathbb{R}}\|\nabla u(s)\|_{(\tilde{q}, \infty)} .
$$


We also defined by $\mathbf{Y}$ the space of vector functions $\left\{\mathbf{u} \in B C\left(\mathbb{R} ; \mathbf{L}_{\sigma}^{(r, \infty)}(\Omega)\right): \nabla \mathbf{u} \in B C\left(\mathbb{R} ; \mathbf{L}^{(q, \infty)}(\Omega)\right)^{n}\right\}$ with the norm $\|\cdot\|_{Y}$ defined as

$$
\|\mathbf{u}\|_{Y} \equiv \sup _{s \in \mathbb{R}}\|\mathbf{u}(s)\|_{(r, \infty)}+\sup _{s \in \mathbb{R}}\|\nabla \mathbf{u}(s)\|_{(q, \infty)} .
$$

$X$ and $\mathbf{Y}$ are Banach spaces. We define the following operators $\mathbf{F}_{1}$ and $G$ on $\mathbf{Y} \times \mathbf{Y}$ and $\mathbf{Y} \times X$, respectively, by

$$
\begin{aligned}
\mathbf{F}_{1}(\mathbf{u}, \mathbf{v})(t) & =-\int_{-\infty}^{t} e^{-(t-s) \mathbf{A}} P[(\mathbf{u} \cdot \nabla) \mathbf{v}](s) d s \\
G(\mathbf{u}, \theta)(t) & =-\int_{-\infty}^{t} e^{-(t-s) B}(\mathbf{u} \cdot \nabla) \theta(s) d s
\end{aligned}
$$

\subsection{Proof of Theorem 2.2}

We construct a periodic solution of Problem (2.8)-(2.9) according to the following scheme:

$$
\mathbf{u}_{m+1}(t)=\mathbf{F}\left(\mathbf{u}_{m}, \theta_{m}\right)(t), \quad \theta_{m+1}(t)=\theta_{0}(t)+G\left(\mathbf{u}_{m}, \theta_{m}\right)(t),
$$

where

$$
\begin{aligned}
\mathbf{u}_{0}(t) & =\int_{-\infty}^{t} e^{-(t-s) \mathbf{A}} P\left(\theta_{0} \mathbf{g}\right) d s, \theta_{0}(t)=\int_{-\infty}^{t} e^{-(t-s) B} f d s, \\
\mathbf{F}\left(\mathbf{u}_{m}, \theta_{m}\right)(t) & =\mathbf{F}_{1}\left(\mathbf{u}_{m}, \mathbf{u}_{m}\right)(t)+\int_{-\infty}^{t} e^{-(t-s) \mathbf{A}}\left\{P\left(\mathbf{g} \theta_{m}\right)\right\} d s, \\
G\left(\mathbf{u}_{m}, \theta_{m}\right)(t) & =-\int_{-\infty}^{t} e^{-(t-s) B}\left(\mathbf{u}_{m} \cdot \nabla\right) \theta_{m}(s) d s .
\end{aligned}
$$

Remark 3.5 In (1.1), when $\boldsymbol{\Psi}$ is not zero, in the scheme above we consider $\mathbf{u}_{0}(t)=\int_{-\infty}^{t} e^{-(t-s) \mathbf{A}} P(\mathbf{\Psi})(s) d s$ and $\mathbf{u}_{m+1}=\mathbf{u}_{0}(t)+\mathbf{F}\left(\mathbf{u}_{m}, \theta_{m}\right)(t)$.

Let us first obtain some estimates for approximations above. We shall need the following lemmas.

Lemma 3.6 Let $r, \tilde{r}, q$ and $\tilde{q}$ be as Theorem 2.2. Then, we have

$$
\begin{aligned}
\sup _{s \in \mathbb{R}}\left\|\mathbf{F}_{1}(\mathbf{u}, \mathbf{v})\right\|_{(r, \infty)} & \leq c_{1} \sup _{s \in \mathbb{R}}\|\mathbf{u}(s)\|_{(r, \infty)}\left(\sup _{s \in \mathbb{R}}\|\mathbf{v}(s)\|_{(r, \infty)}+\sup _{s \in \mathbb{R}}\|\nabla \mathbf{v}(s)\|_{(q, \infty)}\right), \\
\sup _{s \in \mathbb{R}}\left\|\nabla \mathbf{F}_{1}(\mathbf{u}, \mathbf{v})\right\|_{(q, \infty)} & \leq c_{1} \sup _{s \in \mathbb{R}}\|\nabla \mathbf{v}(s)\|_{(q, \infty)}\left(\sup _{s \in \mathbb{R}}\|\mathbf{u}(s)\|_{(r, \infty)}+\sup _{s \in \mathbb{R}}\|\nabla \mathbf{u}(s)\|_{(q, \infty)}\right), \\
\sup _{s \in \mathbb{R}}\|G(\mathbf{u}, \theta)\|_{(\tilde{r}, \infty)} & \leq c_{2} \sup _{s \in \mathbb{R}}\|\mathbf{u}(s)\|_{(r, \infty)}\left(\sup _{s \in \mathbb{R}}\|\theta(s)\|_{(\tilde{r}, \infty)}+\sup _{s \in \mathbb{R}}\|\nabla \theta(s)\|_{(\tilde{q}, \infty)}\right), \\
\sup _{s \in \mathbb{R}}\|\nabla G(\mathbf{u}, \theta)\|_{(\tilde{q}, \infty)} & \leq c_{2} \sup _{s \in \mathbb{R}}\|\nabla \theta(s)\|_{(\tilde{q}, \infty)}\left(\sup _{s \in \mathbb{R}}\|\mathbf{u}(s)\|_{(r, \infty)}+\sup _{s \in \mathbb{R}}\|\nabla \mathbf{u}(s)\|_{(q, \infty)}\right),
\end{aligned}
$$

for every $\mathbf{u}, \mathbf{v} \in \mathbf{Y}, \theta \in X$, where $c_{1}=c_{1}(n, r, q), c_{2}=c_{2}(n, r, q, \tilde{r}, \tilde{q})$. 
Proof. The proof is an application of Lemma 3.2. In fact,

$$
G(\mathbf{u}, \theta)(t)=-\int_{-\infty}^{t-1} e^{-(t-s) B}(\mathbf{u} \cdot \nabla) \theta(s) d s-\int_{t-1}^{t} e^{-(t-s) B}(\mathbf{u} \cdot \nabla) \theta(s) d s=G_{1}(t)+G_{2}(t) .
$$

Then for all $\psi \in C_{0}^{\infty}$ and for all $t \in \mathbb{R}$, we have

$$
\begin{aligned}
\left|\left(G_{1}(t), \psi\right)\right| & \leq \int_{-\infty}^{t-1}\left\|\nabla e^{-(t-s) B} \psi\right\|_{\left((r \tilde{r} /(r+\tilde{r}))^{\prime}, 1\right)}\|\theta \mathbf{u}\|_{(r \tilde{r} /(r+\tilde{r}), \infty)} d s \\
& \leq c \sup _{s \in \mathbb{R}}\|\theta(s)\|_{(\tilde{r}, \infty)} \sup _{s \in \mathbb{R}}\|\mathbf{u}(s)\|_{(r, \infty)} \int_{-\infty}^{t-1}(t-s)^{-n / 2 r-1 / 2}\|\psi\|_{\left(\tilde{r}^{\prime}, 1\right)} .
\end{aligned}
$$

Hence, by duality, for all $t \in \mathbb{R},\left\|G_{1}(t)\right\|_{(\tilde{r}, \infty)} \leq c \sup _{s \in \mathbb{R}}\|\theta(s)\|_{(\tilde{r}, \infty)} \sup _{s \in \mathbb{R}}\|\mathbf{u}(s)\|_{(r, \infty)}$.

$$
\begin{aligned}
\left\|G_{2}(t)\right\|_{(\tilde{r}, \infty)} & \leq \int_{t-1}^{t}(t-s)^{-n / 2(1 / r+1 / \tilde{q}-1 / \tilde{r})}\|\mathbf{u}(s)\|_{(r, \infty)}\|\nabla \theta(s)\|_{(\tilde{q}, \infty)} d s \\
& \leq c \sup _{s \in \mathbb{R}}\|\mathbf{u}(s)\|_{(r, \infty)} \sup _{s \in \mathbb{R}}\|\nabla \theta(s)\|_{(\tilde{q}, \infty)} .
\end{aligned}
$$

Now, using Lemma 3.2 and Lemma 3.4 (for $d=\infty$ ), we get

$$
\begin{aligned}
\|\nabla G(\mathbf{u}, \theta)\|_{(\tilde{q}, \infty)} & \leq \int_{-\infty}^{t-1}\left\|\nabla e^{-(t-s) B}(\mathbf{u} \cdot \nabla) \theta(s)\right\|_{(\tilde{q}, \infty)} d s \\
& +\int_{t-1}^{t}\left\|\nabla e^{-(t-s) B}[(\mathbf{u} \cdot \nabla) \theta](s)\right\|_{(\tilde{q}, \infty)} d s \\
& \leq c \sup _{s \in \mathbb{R}}\|\mathbf{u}(s)\|_{(r, \infty)} \sup _{s \in \mathbb{R}}\|\nabla \theta(s)\|_{(\tilde{q}, \infty)} \int_{-\infty}^{t-1}(t-s)^{-n / 2 r-1 / 2} d s \\
& +c \sup _{s \in \mathbb{R}}\|\mathbf{u}(s)\|_{\left(q^{*}, \infty\right)} \sup _{s \in \mathbb{R}}\|\nabla \theta(s)\|_{(\tilde{q}, \infty)} \int_{t-1}^{t}(t-s)^{-n / 2 q} d s \\
& \left.\leq c \sup _{s \in \mathbb{R}}\|\mathbf{u}(s)\|_{(r, \infty)}+\sup _{s \in \mathbb{R}}\|\nabla \mathbf{u}(s)\|_{(q, \infty)}\right) \sup _{s \in \mathbb{R}}\|\nabla \theta(s)\|_{(\tilde{q}, \infty)},
\end{aligned}
$$

for all $t \in \mathbb{R}$ and $c=c(n, r, q)$. This complete the proof of the last two estimates of lemma. The first two estimates are obtained similarly.

Lemma 3.7 Let $\theta_{0}$ be defined as in (3.16). Then $\theta_{0} \in X$.

Proof. If $f$ satisfies (2.10), then using Lemma 3.2 we obtain

$$
\begin{aligned}
\left\|\theta_{0}(t)\right\|_{(\tilde{r}, \infty)} & \leq c \sup _{s \in \mathbb{R}}\|f(s)\|_{(\tilde{p}, \infty)} \int_{-\infty}^{t-1}(t-s)^{-n / 2(1 / \tilde{p}-1 / \tilde{r})} d s \\
& +c \sup _{s \in \mathbb{R}}\|f(s)\|_{(\tilde{l}, \infty)} \int_{t-1}^{t}(t-s)^{-n / 2(1 / \tilde{l}-1 / \tilde{r})} d s .
\end{aligned}
$$

This is valid for all $t \in \mathbb{R}$. The constant $c=c(n, \tilde{r}, \tilde{p}, \tilde{l})$. From $(2.10)$, that is, $1 / \tilde{r}+2 / n<1 / \tilde{p}$ and $1 / \tilde{l}<2 / n+1 / \tilde{r}$, we conclude that each integral above is finite and consequently, $\left\|\theta_{0}(t)\right\|_{(\tilde{r}, \infty)} \leq$ 
$c_{1} \sup _{s \in \mathbb{R}}\|f(s)\|_{(\tilde{p}, \infty)}+c_{2} \sup _{s \in \mathbb{R}}\|f(s)\|_{(\tilde{l}, \infty)}$, where $c_{1}=c(n, \tilde{p}, \tilde{r})$ and $c_{2}=c(n, \tilde{l}, \tilde{r})$. A similar analysis proves that

$$
\begin{aligned}
\left\|\nabla \theta_{0}(t)\right\|_{(\tilde{q}, \infty)} & \leq \widetilde{c}_{1} \sup _{s \in \mathbb{R}}\|f(s)\|_{(\tilde{p}, \infty)} \int_{-\infty}^{t-1}(t-s)^{-n / 2(1 / \tilde{p}-1 / \tilde{q})-1 / 2} d s \\
& +\widetilde{c}_{2} \sup _{s \in \mathbb{R}}\|f(s)\|_{(\tilde{l}, \infty)} \int_{t-1}^{t}(t-s)^{-n / 2(1 / \tilde{l}-1 / \tilde{q})-1 / 2} d s,
\end{aligned}
$$

for all $t \in \mathbb{R}$ and $\widetilde{c}_{1}=c(n, \widetilde{p}, \widetilde{q})$ and $\widetilde{c}_{2}=c(n, \tilde{l}, \widetilde{q})$. As $1 / \tilde{p}>1 / \tilde{r}+2 / n>1 / n+1 / \tilde{q}$ and $1 / \tilde{l}<1 / \tilde{q}+1 / n$, the two integrals above converge.

Now, if $n=3$, the previous analysis is wrong because it will be necessary $3 / 2(1 / \tilde{p}-1 / \tilde{r})>1$, with $\tilde{p}>1$ and this is not possible. Consequently, we assume a new condition; in fact, if $f$ satisfies (2.11), using the following estimate (which is a consequence of the analytic properties of the semigroup):

$$
\left\|B^{\delta} e^{-t B} \mathbf{a}\right\|_{(\tilde{p}, \infty)} \leq C t^{-\delta}\|\mathbf{a}\|_{(\tilde{p}, \infty)}, \forall \mathbf{a} \in L^{(\tilde{p}, \infty)}, t>0, c=c(\tilde{p}, \delta), \delta \geq 0,
$$

and using Lemma 3.2, we obtain

$$
\begin{aligned}
& \left\|\theta_{0}(t)\right\|_{(\tilde{r}, \infty)} \leq \int_{-\infty}^{t-1}\left\|e^{-(t-s) B} B^{\delta} h(s)\right\|_{(\tilde{r}, \infty)} d s+\int_{t-1}^{t}\left\|e^{-(t-s) B} f(s)\right\|_{(\tilde{r}, \infty)} d s \\
& \leq c \int_{-\infty}^{t-1}(t-s)^{-3 / 2(1 / \tilde{p}-1 / \tilde{r})}\left\|B^{\delta} e^{-(t-s) B / 2} h(s)\right\|_{(\tilde{p}, \infty)} d s+c \int_{t-1}^{t}(t-s)^{-3 / 2(1 / \tilde{l}-1 / \tilde{r})}\|f(s)\|_{(\tilde{l}, \infty)} d s \\
& \leq c \int_{-\infty}^{t-1}(t-s)^{-3 / 2(1 / \tilde{p}-1 / \tilde{r})-\delta}\|h(s)\|_{(\tilde{p}, \infty)} d s+\sup _{s \in \mathbb{R}}\|f(s)\|_{(\tilde{l}, \infty)} \int_{t-1}^{t}(t-s)^{-3 / 2(1 / \tilde{l}-1 / \tilde{r})} d s \\
& \leq c\left(\sup _{s \in \mathbb{R}}\|h(s)\|_{(\tilde{p}, \infty)}+\sup _{s \in \mathbb{R}}\|f(s)\|_{(\tilde{l}, \infty)}\right),
\end{aligned}
$$

for all $t \in \mathbb{R}$ with $c=c(n, \tilde{r}, \tilde{p}, \tilde{l}, \delta)$. A similar estimate can be obtained for $\left\|\nabla \theta_{0}\right\|_{(\tilde{q}, \infty)}, \quad(n=3)$. This proves the lemma.

Now we will estimate the terms $\mathbf{F}\left(\mathbf{u}_{m}, \theta_{m}\right)$ and $G\left(\mathbf{u}_{m}, \theta_{m}\right)$. We start with the following lemma

Lemma 3.8 The terms $\left\|\mathbf{F}\left(\mathbf{u}_{m}, \theta_{m}\right)\right\|_{Y},\left\|G\left(\mathbf{u}_{m}, \theta_{m}\right)\right\|_{X}$ given by (3.17),(3.18) satisfy

$$
\begin{aligned}
\left\|\mathbf{F}\left(\mathbf{u}_{m}, \theta_{m}\right)\right\|_{Y} & \leq 2 c_{1}\left\|\mathbf{u}_{m}\right\|_{Y}^{2}+c_{3}\left\|\theta_{m}\right\|_{X}, \\
\left\|G\left(\mathbf{u}_{m}, \theta_{m}\right)\right\|_{X} & \leq 2 c_{2}\left\|\mathbf{u}_{m}\right\|_{Y}\left\|\theta_{m}\right\|_{X},
\end{aligned}
$$

where $c_{1}, c_{2}$ are as in Lemma 3.6 and $c_{3}$ depends on $\mathbf{g}$ but is independent of $m$.

Proof. We will prove that

$$
\left\|\int_{-\infty}^{t} e^{-(t-s) \mathbf{A}} P\left(\mathbf{g} \theta_{m}\right)(s) d s\right\|_{Y} \leq c_{3}\left\|\theta_{m}\right\|_{X}
$$


In fact,

$$
\begin{aligned}
\left\|\int_{-\infty}^{t} e^{-(t-s) \mathbf{A}} P\left(\mathbf{g} \theta_{m}\right)(s) d s\right\|_{(r, \infty)} & \leq \int_{-\infty}^{t-1}\left\|e^{-(t-s) \mathbf{A}} P\left(\mathbf{g} \theta_{m}\right)(s)\right\|_{(r, \infty)} d s \\
& +\int_{t-1}^{t}\left\|e^{-(t-s) \mathbf{A}} P\left(\mathbf{g} \theta_{m}\right)(s)\right\|_{(r, \infty)} d s .
\end{aligned}
$$

Note that

$$
\int_{-\infty}^{t-1}\left\|e^{-(t-s) \mathbf{A}} P\left(\mathbf{g} \theta_{m}\right)(s)\right\|_{(r, \infty)} d s \leq c\|\mathbf{g}\|_{(a, \infty)} \sup _{s \in \mathbb{R}}\left\|\theta_{m}(s)\right\|_{(\tilde{r}, \infty)} \int_{-\infty}^{t-1}(t-s)^{\gamma} d s
$$

where $\gamma=-n / 2(1 / a+1 / \tilde{r}-1 / r)$. As $1 / a>2 / n-1 / \tilde{r}+1 / r$, the last integral converges. Moreover, for $\xi=-n / 2(1 / b+1 / \tilde{r}-1 / r)$ we have

$$
\int_{t-1}^{t}\left\|e^{-(t-s) \mathbf{A}} P\left(\mathbf{g} \theta_{m}\right)(s)\right\|_{(r, \infty)} d s \leq c\|\mathbf{g}\|_{(b, \infty)} \sup _{s \in \mathbb{R}}\left\|\theta_{m}(s)\right\|_{(\tilde{r}, \infty)} \int_{t-1}^{t}(t-s)^{\xi} d s,
$$

By Assumption 1, $\mathbf{g} \in \mathbf{L}^{(b, \infty)}(\Omega)$ with $b>1$ and $1 / b<1 / n+1 / q-1 / \tilde{r}$. As $r<n, n / 2<q$, we have that $1 / r+1 / n>2 / n>1 / q$, and therefore $1 / b<1 / n+1 / q-1 / \tilde{r}<1 / n+1 / n=2 / n$ which implies that $1 / b<2 / n+1 / r-1 / \tilde{r}$ and thus, the last integral converges.

On the other hand

$$
\begin{aligned}
\left\|\nabla \int_{-\infty}^{t} e^{-(t-s) \mathbf{A}} P\left(\mathbf{g} \theta_{m}\right)(s)\right\|_{(q, \infty)} d s & \leq \int_{-\infty}^{t-1}\left\|\nabla e^{-(t-s) \mathbf{A}} P\left(\mathbf{g} \theta_{m}\right)(s)\right\|_{(q, \infty)} d s \\
& +\int_{t-1}^{t}\left\|\nabla e^{-(t-s) \mathbf{A}} P\left(\mathbf{g} \theta_{m}\right)(s)\right\|_{(q, \infty)} d s
\end{aligned}
$$

where we estimate the first integral as

$$
\int_{-\infty}^{t-1}\left\|\nabla e^{-(t-s) \mathbf{A}} P\left(\mathbf{g} \theta_{m}\right)(s)\right\|_{(q, \infty)} d s \leq c\|\mathbf{g}\|_{(a, \infty)} \sup _{s \in \mathbb{R}}\left\|\theta_{m}(s)\right\|_{(\tilde{r}, \infty)} \int_{-\infty}^{t-1}(t-s)^{\zeta} d s,
$$

for $\zeta=-n / 2(1 / a+1 / \tilde{r}-1 / q)-1 / 2$. As $n<q$ and $1 / \tilde{r}-1 / r>2 / n-1 / a$, we conclude that $1 / a+1 / \tilde{r}-1 / q>2 / n+1 / r-1 / q>1 / n+1 / r>1 / n$. Hence the last integral converges. Analogously, we can show that

$$
\int_{t-1}^{t}\left\|\nabla e^{-(t-s) \mathbf{A}} P\left(\mathbf{g} \theta_{m}\right)(s)\right\|_{(q, \infty)} d s \leq c\|\mathbf{g}\|_{(b, \infty)} \sup _{s \in \mathbb{R}}\left\|\theta_{m}(s)\right\|_{(\tilde{r}, \infty)} \int_{t-1}^{t}(t-s)^{\xi} d s,
$$

with $\xi=-n / 2(1 / b+1 / \widetilde{r}-1 / q)-1 / 2$ and thus the integral converges. Hence, we prove inequality (3.21) with $c_{3}=c\left(\|\mathbf{g}\|_{(b, \infty)}+\|\mathbf{g}\|_{(a, \infty)}\right)$ and $c=c(n, a, b, r, q, \widetilde{r}, \widetilde{q})$ independent of $m$. Therefore, from Lemma 3.6 and estimate (3.21), we obtain (3.19). Inequality (3.20) is obtained applying directly the last two inequalities of Lemma 3.6.

After these Lemmas we back to the proof of Theorem 2.2. 


\section{Existence.}

From scheme (3.15), Lemma 3.7 and Lemma 3.8, we obtain:

$$
\begin{aligned}
\left\|\mathbf{u}_{m+1}\right\|_{Y} & \leq 2 c_{1}\left\|\mathbf{u}_{m}\right\|_{Y}^{2}+c_{3}\left\|\theta_{m}\right\|_{X}, \\
\left\|\theta_{m+1}\right\|_{X} & \leq\left\|\theta_{0}\right\|_{X}+2 c_{2}\left\|\mathbf{u}_{m}\right\|_{Y}\left\|\theta_{m}\right\|_{X} .
\end{aligned}
$$

Let $a_{m}=\max \left\{\left\|\mathbf{u}_{m}\right\|_{Y},\left\|\theta_{m}\right\|_{X}\right\}, m=1,2, \ldots \quad a_{0}=\left\|\theta_{0}\right\|_{X}$. Therefore, from (3.22) and (3.23) it follows that $a_{m+1} \leq a_{0}+\widetilde{c} a_{m}^{2}+c_{3} a_{m}, \widetilde{c}=\max \left(2 c_{1}, 2 c_{2}\right)$. Hence, if

$$
c_{3}<1, \quad 4 a_{0} \widetilde{c}<\left(1-c_{3}\right)^{2},
$$

then, the sequence $\left\{a_{m}\right\}_{m=0}^{\infty}$ is bounded and

$$
a_{m} \leq \frac{\left(1-c_{3}\right)-\sqrt{\left(1-c_{3}\right)^{2}-4 \widetilde{c} a_{0}}}{2 \widetilde{c}} \equiv k, \forall m=0,1, \ldots \Rightarrow a_{m} \leq k<\frac{1}{2 \widetilde{c}} .
$$

From now on, we assume (3.24) (Note that this condition implies a small condition for $f$ ). Making $\mathbf{w}_{m}=\mathbf{u}_{m}-\mathbf{u}_{m-1}\left(\mathbf{u}_{-1} \equiv 0\right), \Theta_{m}=\theta_{m}-\theta_{m-1},\left(\theta_{-1} \equiv 0\right)$, we have

$$
\begin{aligned}
& \mathbf{w}_{m+1}(t)=\mathbf{F}_{1}\left(\mathbf{w}_{m}, \mathbf{u}_{m}\right)(t)+\mathbf{F}_{1}\left(\mathbf{u}_{m-1}, \mathbf{w}_{m}\right)(t)+\int_{-\infty}^{t} e^{-(t-s) \mathbf{A}} P\left(\mathbf{g} \Theta_{m}\right)(s) d s, \\
& \Theta_{m+1}(t)=G\left(\mathbf{w}_{m}, \theta_{m}\right)(t)+G\left(\mathbf{u}_{m-1}, \Theta_{m}\right)(t) .
\end{aligned}
$$

This equality implies that

$$
\begin{aligned}
\left\|\mathbf{w}_{m+1}\right\|_{Y} & \leq 2 c_{1}\left(\left\|\mathbf{w}_{m}\right\|_{Y}\left\|\mathbf{u}_{m}\right\|_{Y}+\left\|\mathbf{u}_{m-1}\right\|_{Y}\left\|\mathbf{w}_{m}\right\|_{Y}\right)+c_{3}\left\|\Theta_{m}\right\|_{X} \\
& \leq 2 \widetilde{c} k\left(\left\|\mathbf{w}_{m}\right\|_{Y}+\left\|\Theta_{m}\right\|_{X}\right)
\end{aligned}
$$

provided $c_{3} \leq \widetilde{c} k$ (this condition and (3.24) imply a small condition for the field $\mathbf{g}$ in the norms $\|\cdot\|_{(a, \infty)}$ and $\left.\|\cdot\|_{(b, \infty)}\right)$. Moreover,

$$
\left\|\Theta_{m+1}\right\|_{X} \leq 2 c_{2}\left\|\mathbf{w}_{m}\right\|_{Y}\left\|\theta_{m}\right\|_{X}+2 c_{2}\left\|\mathbf{u}_{m-1}\right\|_{Y}\left\|\Theta_{m}\right\|_{X} \leq 2 c_{2} k\left(\left\|\mathbf{w}_{m}\right\|_{Y}+\left\|\Theta_{m}\right\|_{X}\right)
$$

From (3.26)-(3.27), we obtain

$$
\begin{aligned}
\max \left\{\left\|\mathbf{w}_{m+1}\right\|_{Y},\left\|\Theta_{m+1}\right\|_{X}\right\} & \leq 2 \widetilde{c} k \max \left\{\left\|\mathbf{w}_{m}\right\|_{Y},\|\Theta\|_{X}\right\} \leq \ldots \\
& \leq(2 \widetilde{c} k)^{m+1} a_{0}, \quad \forall m=0,1, \ldots
\end{aligned}
$$

Note that $\mathbf{u}_{m}(t)=\sum_{j=0}^{m} \mathbf{w}_{j}(t), \theta_{m}(t)=\sum_{j=0}^{m} \Theta_{j}(t)$. Since $2 \widetilde{c} k<1$ (by (3.25)), from (3.28) we can conclude that there exist functions $\mathbf{u} \in \mathbf{Y}, \theta \in X$ such that when $m \longrightarrow \infty, \mathbf{u}_{m} \longrightarrow \mathbf{u}$ in $\mathbf{Y}$, $\theta_{m} \longrightarrow \theta$ in $X$. Note that

$$
\begin{array}{r}
\left\|\mathbf{F}_{1}\left(\mathbf{u}_{m}, \mathbf{u}_{m}\right)(t)-\mathbf{F}_{1}(\mathbf{u}, \mathbf{u})(t)\right\|_{Y} \leq\left\|\mathbf{F}_{1}\left(\mathbf{u}_{m}-\mathbf{u}, \mathbf{u}_{m}\right)(t)\right\|_{Y}+\left\|\mathbf{F}_{1}\left(\mathbf{u}, \mathbf{u}_{m}-\mathbf{u}\right)(t)\right\|_{Y} \\
\leq 2 c_{1}\left\|\mathbf{u}_{m}-\mathbf{u}\right\|_{Y}\left\|\mathbf{u}_{m}\right\|_{Y}+2 c_{1}\|\mathbf{u}\|_{Y}\left\|\mathbf{u}_{m}-\mathbf{u}\right\|_{Y}<\left\|\mathbf{u}_{m}-\mathbf{u}\right\|_{Y}, \quad \forall m
\end{array}
$$


Then, in $\mathbf{Y}$

$$
\mathbf{F}_{1}\left(\mathbf{u}_{m}, \mathbf{u}_{m}\right)(t) \longrightarrow \mathbf{F}_{1}(\mathbf{u}, \mathbf{u})(t)
$$

Analogously,

$$
\begin{aligned}
\left\|G\left(\mathbf{u}_{m}, \theta_{m}\right)(t)-G(\mathbf{u}, \theta)(t)\right\|_{X} & \leq 2 c_{2}\left\|\mathbf{u}_{m}-\mathbf{u}\right\|_{Y}\left\|\theta_{m}\right\|_{X}+2 c_{2}\|\mathbf{u}\|_{Y}\left\|\theta_{m}-\theta\right\|_{X} \\
& <\left\|\mathbf{u}_{m}-\mathbf{u}\right\|_{Y}+\left\|\theta_{m}-\theta\right\|_{X}, \forall m .
\end{aligned}
$$

Then, in $X$

$$
G\left(\mathbf{u}_{m}, \theta_{m}\right)(t) \longrightarrow G(\mathbf{u}, \theta)(t)
$$

Finally, when $m \longrightarrow \infty$,

$$
\left\|-\int_{-\infty}^{t} e^{-(t-s) \mathbf{A}} P\left(\mathbf{g}\left(\theta_{m}-\theta\right)\right)(s) d s\right\|_{Y} \leq c_{3}\left\|\theta_{m}-\theta\right\|_{X} \longrightarrow 0
$$

From (3.29), (3.30) and (3.31) we conclude that $(\mathbf{u}, \theta)$ is a solution of the system of integral equations (2.8)-(2.9).

\section{Periodicity.}

Being $f$ a periodic function with period $\tau>0$, the functions $\mathbf{u}_{m}$ and $\theta_{m}$ are also periodic with the same period $\tau$ for all $m=0,1,2, \ldots$ Consequently, the limit $(\mathbf{u}, \theta)$ is periodic with period $\tau$.

\section{Uniqueness.}

Suppose that $\left(\mathbf{u}_{1}, \theta_{1}\right)$ is another solution of (2.8)-(2.9), such that $\left\|\mathbf{u}_{1}\right\|_{Y} \leq k,\left\|\theta_{1}\right\|_{X} \leq k$, being $k$ the constant of (3.25). Working as the proof of existence we get

$$
\begin{aligned}
\left\|\theta-\theta_{1}\right\|_{X} & \leq 2 c_{2} k\left\|\mathbf{u}-\mathbf{u}_{1}\right\|_{Y}+2 c_{2} k\left\|\theta-\theta_{1}\right\|_{X}, \\
\left\|\mathbf{u}-\mathbf{u}_{1}\right\|_{Y} & \leq c_{3}\left\|\theta-\theta_{1}\right\|_{X}+2 c_{1} k\left\|\mathbf{u}-\mathbf{u}_{1}\right\|_{Y} .
\end{aligned}
$$

Hence, if $M \equiv \max \left\{\left\|\mathbf{u}-\mathbf{u}_{1}\right\|_{Y},\left\|\theta-\theta_{1}\right\|_{X}\right\}$, we have $M \leq 2 \widetilde{c} k M$, because $c_{3} \leq \widetilde{c} k$, which implies that $\theta=\theta_{1}$ and $\mathbf{u}=\mathbf{u}_{1}$. Hence, the proof of Theorem 2.2 is finished.

\subsection{Strong Solution. Proof of Theorem 2.3.}

In this subsection we prove that if $f$ and $\mathbf{g}$ satisfy adequate regularity conditions, then the periodic solution $(\mathbf{u}, \theta)$ constructed in Theorem 2.2 is also a solution of the differential system (1.1)-(1.5). For the proof of Theorem 2.3, we need a result about local existence of strong solutions for the initial boundary value problem associated to (1.1)-(1.5) that will be presented as Theorem 3.10. This result follows the arguments of Kato [10]. Let us first give the definition of strong solution for the initial value problem (1.1)-(1.5). 
Definition 3.9 Let $\mathbf{a} \in \mathbf{L}_{\sigma}^{(n, \infty)}(\Omega), b \in L^{(n, \infty)}(\Omega), n<r^{*}<\infty, f$ being Hölder continuous with values in $L^{\left(r^{*}, \infty\right)}(\Omega)$ and $\mathbf{g} \in \mathbf{L}^{\left(r^{*}, \infty\right)}(\Omega)$. A pair $(\mathbf{v}, w)$ defined on $\left(t_{0}, t_{1}\right) \times \Omega$ is called a $\mathbf{s t r o n g}$ solution of (1.1)-(1.5) of class $S_{r^{*}}\left(t_{0}, t_{1}\right)$, with initial value $(\mathbf{a}, b)$ if

1. $\mathbf{v} \in B C_{w}\left(\left[t_{0}, t_{1}\right) ; \mathbf{L}_{\sigma}^{(n, \infty)}(\Omega)\right) \cap C^{1}\left(\left(t_{0}, t_{1}\right) ; \mathbf{L}_{\sigma}^{r^{*}}(\Omega)\right)$, $w \in B C_{w}\left(\left[t_{0}, t_{1}\right) ; L^{(n, \infty)}(\Omega)\right) \cap C^{1}\left(\left(t_{0}, t_{1}\right) ; L^{r^{*}}(\Omega)\right)$,

2. $\mathbf{A} \mathbf{v} \in C\left(\left(t_{0}, t_{1}\right) ; \mathbf{L}_{\sigma}^{r^{*}}(\Omega)\right), B w \in C\left(\left(t_{0}, t_{1}\right) ; L^{r^{*}}(\Omega)\right), t_{0}<t<t_{1}$,

3. $\mathbf{v}_{t}+\mathbf{A} \mathbf{v}+P[(\mathbf{v} \cdot \nabla) \mathbf{v}]=P(w \mathbf{g})$ in $\mathbf{L}_{\sigma}^{r^{*}}(\Omega)$, for $x \in \Omega, t_{0}<t<t_{1}$,

4. $w_{t}+B w+(\mathbf{v} \cdot \nabla w)=f$ in $L^{r^{*}}(\Omega)$, for $x \in \Omega, t_{0}<t<t_{1}$,

where $B C_{w}$ denotes the class of bounded and weakly-* continuous functions, together with $\lim _{t \rightarrow t_{0}^{+}}(\mathbf{v}(t), \boldsymbol{\phi})=(\mathbf{a}, \boldsymbol{\phi}), \lim _{t \rightarrow t_{0}^{+}}(w(t), \varphi)=(b, \varphi)$, for all $\boldsymbol{\phi} \in \mathbf{L}_{\sigma}^{(n /(n-1), 1)}(\Omega), \varphi \in L^{(n /(n-1), 1)}(\Omega)$.

Our result about the local existence of strong solutions now reads

Theorem 3.10 (i) (Existence). Let $n / 2<q<n$ and $1<l<\infty$ be such that $1 / q<1 / l<$ $1 / q+1 / n$. Supposed that $\mathbf{a} \in \mathbf{L}_{\sigma}^{(n, \infty)}(\Omega) \cap \mathbf{L}_{\sigma}^{\left(q^{*}, \infty\right)}(\Omega), b \in L^{(n, \infty)}(\Omega) \cap L^{\left(q^{*}, \infty\right)}(\Omega)$, where $q^{*}=n q /(n-q), f \in B C\left(\mathbb{R} ; L^{(l, \infty)}(\Omega)\right)$ being Hölder continuous with values in $L^{\left(r^{*}, \infty\right)}(\Omega)$, $\mathbf{g} \in \mathbf{L}^{(b, \infty)}(\Omega) \cap \mathbf{L}^{\left(r^{*}, \infty\right)}(\Omega)$ with $b>n / 2$ and $n<r^{*}<q^{*}$. Then, there exists $T \in(0,1]$ such that for all $t_{0} \in \mathbb{R}$ there exists a strong solution of class $S_{r^{*}}\left(t_{0}, t_{0}+T\right)$ of problem (1.1)-(1.5) at $\left(t_{0}, t_{0}+T\right)$ with initial value $\mathbf{v}\left(t_{0}\right)=\mathbf{a}, w\left(t_{0}\right)=b$. Moreover, the solution satisfies $\mathbf{v} \in B C\left(\left(t_{0}, t_{0}+T\right) ; \mathbf{L}_{\sigma}^{\left(q^{*}, \infty\right)}(\Omega)\right), w \in B C\left(\left(t_{0}, t_{0}+T\right) ; L^{\left(q^{*}, \infty\right)}(\Omega)\right)$, with

$$
\sup _{t_{0}<t<t_{0}+T}\|\mathbf{v}(t)\|_{\left(q^{*}, \infty\right)} \leq C_{1}, \sup _{t_{0}<t<t_{0}+T}\|w(t)\|_{\left(q^{*}, \infty\right)} \leq C_{2},
$$

where $C_{1}, C_{2}$ are independent of $t_{0}$. Here $T$ is estimated as

$$
T \equiv\left[\frac{\tilde{k}}{c_{1} \max \left\{\|\mathbf{a}\|_{(n / \alpha, \infty)},\|b\|_{(n / \alpha, \infty)}+\|f\|_{B C\left(\mathbb{R} ; L^{(l, \infty)}\right)}\right\}}\right]^{\frac{2}{1-\alpha}}
$$

with $\tilde{k} / c_{1}=\tilde{k} / c_{1}(n, q, l), \alpha=n / q^{*}$.

(ii) (Uniqueness). There exists a constant $\gamma=\gamma\left(n, r^{*}\right)$ such that any solution $(\mathbf{v}, w)$ in the above class, satisfying

$$
\limsup _{t \rightarrow t_{0}^{+}} t^{n / 2\left(1 / n-1 / r^{*}\right)}\|\mathbf{v}(t)\|_{r^{*}} \leq \gamma, \quad \limsup _{t \rightarrow t_{0}^{+}} t^{n / 2\left(1 / n-1 / r^{*}\right)}\|w(t)\|_{r^{*}} \leq \gamma
$$

is unique. 
Proposition 3.11 Let $n / 2<q<n$ and $1<l<\infty$ be such that $1 / q<1 / l<1 / q+1 / n$. Supposed that $\mathbf{a} \in \mathbf{L}_{\sigma}^{(n, \infty)}(\Omega) \cap \mathbf{L}_{\sigma}^{\left(q^{*}, \infty\right)}(\Omega), b \in L^{(n, \infty)}(\Omega) \cap L^{\left(q^{*}, \infty\right)}(\Omega)$, where $q^{*}=n q /(n-q)$, $f \in B C\left(\mathbb{R} ; L^{(l, \infty)}(\Omega)\right)$ with value in $L^{(n, \infty)}(\Omega), \mathbf{g} \in \mathbf{L}^{(b, \infty)}(\Omega) \cap \mathbf{L}^{(n, \infty)}(\Omega)$ with $b>n / 2$. Then, there exists $T \in(0,1]$ and functions $\mathbf{v}, w$ in the class

$$
(\mathbf{v}, w) \in B C_{w}\left(\left[t_{0}, t_{0}+T\right) ; \mathbf{L}_{\sigma}^{(n, \infty)}(\Omega) \times L^{(n, \infty)}(\Omega)\right)
$$

with

$$
\left\{\begin{aligned}
(\mathbf{v}, w) & \in B C\left(\left(t_{0}, t_{0}+T\right) ; \mathbf{L}^{\left(q^{*}, \infty\right)}(\Omega) \times L^{\left(q^{*}, \infty\right)}(\Omega)\right), \\
\left(t-t_{0}\right)^{1 / 2} \nabla(\mathbf{v}, w) & \in B C_{w}\left(\left(t_{0}, t_{0}+T\right) ; \mathbf{L}^{(n, \infty)}(\Omega) \times L^{(n, \infty)}(\Omega)\right)^{n},
\end{aligned}\right.
$$

such that for all $t_{0} \in \mathbb{R}$ the following equalities are verified in $\mathbf{L}_{\sigma}^{(n, \infty)}(\Omega) \cap \mathbf{L}_{\sigma}^{r^{*}}(\Omega)$ and $L^{(n, \infty)}(\Omega) \cap$ $L^{r^{*}}(\Omega), n<r^{*}<q^{*}$, respectively

$$
\begin{aligned}
& \mathbf{v}(t)=e^{-\left(t-t_{0}\right) \mathbf{A}} \mathbf{a}+\int_{t_{0}}^{t} e^{-(t-s) \mathbf{A}} P(w \mathbf{g})-\int_{t_{0}}^{t} e^{-(t-s) \mathbf{A}} P[(\mathbf{v} \cdot \nabla) \mathbf{v}] d s, \\
& w(t)=e^{-\left(t-t_{0}\right) B} b+\int_{t_{0}}^{t} e^{-(t-s) B} f(s) d s-\int_{t_{0}}^{t} e^{-(t-s) B}(\mathbf{v} \cdot \nabla w)(s) d s .
\end{aligned}
$$

Moreover, the functions $(\mathbf{v}, w)$ satisfy

$$
t^{1 / 4}(\mathbf{v}, w) \in B C\left(\left(t_{0}, t_{0}+T\right) ; \mathbf{L}_{\sigma}^{2 n}(\Omega) \times L^{2 n}(\Omega)\right)
$$

Here $T$ is estimated as (3.33), where $\tilde{k} / c_{1}=\tilde{k} / c_{1}(n, q, l), \alpha=n / q^{*}$.

Proof of Proposition 3.11: Let us construct the solutions of integral equations (3.34)(3.35) according to the following scheme:

$$
\begin{aligned}
\mathbf{v}_{m+1}(t)= & \mathbf{v}_{0}(t)+\int_{t_{0}}^{t} e^{-(t-s) \mathbf{A}} P\left(w_{m} \mathbf{g}\right) d s-\int_{t_{0}}^{t} e^{-(t-s) \mathbf{A}} P\left[\left(\mathbf{v}_{m} \cdot \nabla\right) \mathbf{v}_{m}\right] d s \\
& w_{m+1}(t)=w_{0}(t)+\int_{t_{0}}^{t} e^{-(t-s) B} f d s-\int_{t_{0}}^{t} e^{-(t-s) B}\left(\mathbf{v}_{m} \cdot \nabla\right) w_{m} d s
\end{aligned}
$$

where $\mathbf{v}_{0}(t)=e^{-\left(t-t_{0}\right) \mathbf{A}} \mathbf{a}, w_{0}(t)=e^{-\left(t-t_{0}\right) B} b$.

Since this Lemma only deals with local existence of solutions, we may assume that $0<T \leq 1$. We observe that $\alpha=n / q^{*}, q^{*}=n q /(n-q)$, and as $<2 q$, then $0<\alpha<1$. We will need the following lemmas

Lemma 3.12 The sequences (3.36), (3.37) satisfy the following estimates

$$
\begin{aligned}
& \sup _{t_{0}<t<t_{0}+T}\left(t-t_{0}\right)^{(1-\alpha) / 2}\left\|\mathbf{v}_{m}(t)\right\|_{(n / \alpha, \infty)} \leq K_{m, 1} \\
& \sup _{t_{0}<t<t_{0}+T}\left(t-t_{0}\right)^{(1-\alpha) / 2}\left\|w_{m}(t)\right\|_{(n / \alpha, \infty)} \leq K_{m, 2}, m=0,1, \ldots
\end{aligned}
$$


for some positive constants $K_{m, 1}, K_{m, 2}$ which are independent of $t_{0}$. Moreover, there exists $(\mathbf{v}, w)$ with

$$
\left(t-t_{0}\right)^{(1-\alpha) / 2}(\mathbf{v}(\cdot), w(\cdot)) \in B C\left(\left(t_{0}, t_{0}+T\right) ; \mathbf{L}_{\sigma}^{(n / \alpha, \infty)}(\Omega) \times L^{(n / \alpha, \infty)}(\Omega)\right),
$$

such that

$$
\begin{aligned}
& \lim _{m \rightarrow \infty} \sup _{t_{0}<t<t_{0}+T}\left(t-t_{0}\right)^{(1-\alpha) / 2}\left\|\mathbf{v}_{m}(t)-\mathbf{v}(t)\right\|_{(n / \alpha, \infty)}=0 \\
& \lim _{m \rightarrow \infty} \sup _{t_{0}<t<t_{0}+T}\left(t-t_{0}\right)^{(1-\alpha) / 2} \| w_{m}(t)-\left.w(t)\right|_{(n / \alpha, \infty)}=0 .
\end{aligned}
$$

Proof. The proof is done by induction. In fact, $\left\|\mathbf{v}_{0}(t)\right\|_{(n / \alpha, \infty)} \leq c\|\mathbf{a}\|_{(n / \alpha, \infty)}$ and $\left\|w_{0}(t)\right\|_{(n / \alpha, \infty)} \leq$ $c\|b\|_{(n / \alpha, \infty)}$, for $t_{0}<t<t_{0}+T$, where $c$ is independent of $t_{0}$. Consequently,

$$
\begin{gathered}
\sup _{t_{0}<t<t_{0}+T}\left(t-t_{0}\right)^{(1-\alpha) / 2}\left\|\mathbf{v}_{0}(t)\right\|_{(n / \alpha, \infty)} \leq c T^{(1-\alpha) / 2}\|\mathbf{a}\|_{(n / \alpha, \infty)} \equiv K_{0,1} \\
\sup _{t_{0}<t<t_{0}+T}\left(t-t_{0}\right)^{(1-\alpha) / 2}\left\|w_{0}(t)\right\|_{(n / \alpha, \infty)} \leq c T^{(1-\alpha) / 2}\|b\|_{(n / \alpha, \infty)} \equiv K_{0,2} .
\end{gathered}
$$

Assume (3.38)-(3.39) are true. We will prove (3.38)-(3.39) for the case $m+1$. Note that for all $\phi \in \mathbf{C}_{0, \sigma}^{\infty}$ and all $t_{0}<t<t_{0}+T$, Lemma 3.2 implies

$$
\begin{aligned}
\mid\left(-\int_{t_{0}}^{t} e^{-(t-s) \mathbf{A}} P\left[\left(\mathbf{v}_{m} \cdot \nabla\right) \mathbf{v}_{m}\right]\right. & d s, \boldsymbol{\phi}) \mid \leq \int_{t_{0}}^{t}\left\|\mathbf{v}_{m}(s)\right\|_{(n / \alpha, \infty)}^{2}\left\|\nabla e^{-(t-s) \mathbf{A}} \boldsymbol{\phi}\right\|_{(n /(n-2 \alpha), 1)} \\
\leq & c \int_{t_{0}}^{t}(t-s)^{-\alpha / 2-1 / 2}\left\|\mathbf{v}_{m}(s)\right\|_{(n / \alpha, \infty)}^{2} d s \cdot\|\boldsymbol{\phi}\|_{(n /(n-\alpha), 1)} \\
& \leq c B((1-\alpha) / 2, \alpha) K_{m, 1}^{2}\left(t-t_{0}\right)^{-(1-\alpha) / 2}\|\boldsymbol{\phi}\|_{(n /(n-\alpha), 1)},
\end{aligned}
$$

where $B(\cdot, \cdot)$ denotes the Beta function and $c=c(n, q)$ is independent of $t_{0}$. By duality we have

$$
\left\|\int_{t_{0}}^{t} e^{-(t-s) \mathbf{A}} P\left[\left(\mathbf{v}_{m} \cdot \nabla\right) \mathbf{v}_{m}\right](s) d s\right\|_{(n / \alpha, \infty)} \leq C_{1,1} K_{m, 1}^{2}\left(t-t_{0}\right)^{-(1-\alpha) / 2},
$$

for all $t, t_{0}<t<t_{0}+T$, with $C_{1,1}=C_{1,1}(n, q)$, and moreover

$$
\begin{gathered}
\left\|\int_{t_{0}}^{t} e^{-(t-s) \mathbf{A}} P\left(\mathbf{g} w_{m}\right)(s) d s\right\|_{(n / \alpha, \infty)} \leq c \int_{t_{0}}^{t}(t-s)^{-n / 2 b}\|\mathbf{g}\|_{(b, \infty)}\left\|w_{m}(s)\right\|_{(n / \alpha, \infty)} d s \\
\quad \leq c\left(t-t_{0}\right)^{-(1-\alpha) / 2} K_{m, 2}\|\mathbf{g}\|_{(b, \infty)}\left(t-t_{0}\right)^{1-n / 2 b} B\left(-\frac{n}{2 b}+1, \frac{(1+\alpha)}{2}\right) \\
\leq\left(t-t_{0}\right)^{-(1-\alpha) / 2} C_{2,1} K_{m, 2} T^{1-n / 2 b}
\end{gathered}
$$

being $C_{2,1}=c\|\mathbf{g}\|_{(b, \infty)} B\left(-\frac{n}{2 b}+1, \frac{(1+\alpha)}{2}\right)$. Hence

$$
\sup _{t_{0}<t<t_{0}+T}\left(t-t_{0}\right)^{(1-\alpha) / 2}\left\|\mathbf{v}_{m+1}\right\|_{(n / \alpha, \infty)} \leq K_{0,1}+C_{1,1} K_{m, 1}^{2}+C_{2,1} T^{1-n / 2 b} K_{m, 2} .
$$

Moreover, for all $\phi \in \mathbf{C}_{0}^{\infty}$ and all $t_{0}<t<t_{0}+T$, we have

$$
\begin{aligned}
\mid(- & \left.\int_{t_{0}}^{t} e^{-(t-s) B}\left(\mathbf{v}_{m} \cdot \nabla w_{m}\right)(s) d s, \boldsymbol{\phi}\right)|=| \int_{t_{0}}^{t}\left(w_{m} \otimes \mathbf{v}_{m}(s), \nabla e^{-(t-s) B} \boldsymbol{\phi}\right) d s \mid \\
& \leq \int_{t_{0}}^{t}\left\|\mathbf{v}_{m}(s)\right\|_{(n / \alpha, \infty)}\left\|w_{m}(s)\right\|_{(n / \alpha, \infty)}\left\|\nabla e^{-(t-s) B} \boldsymbol{\phi}\right\|_{(n /(n-2 \alpha), 1)} d s \\
& \leq c \int_{t_{0}}^{t}(t-s)^{-\alpha / 2-1 / 2}\left\|\mathbf{v}_{m}(s)\right\|_{(n / \alpha, \infty)}\left\|w_{m}(s)\right\|_{(n / \alpha, \infty)} \cdot\|\phi\|_{(n /(n-\alpha), 1)} d s \\
& \leq c B((1-\alpha) / 2, \alpha) K_{m, 1} K_{m, 2}\left(t-t_{0}\right)^{-(1-\alpha) / 2}\|\phi\|_{(n /(n-\alpha), 1)} .
\end{aligned}
$$


By duality, for $t_{0}<t<t_{0}+T$, we get

$$
\left\|\int_{t_{0}}^{t} e^{-(t-s) B}\left(\mathbf{v}_{m} \cdot \nabla w_{m}\right)(s) d s\right\|_{(n / \alpha, \infty)} \leq C_{1,2} K_{m, 2} K_{m, 1}\left(t-t_{0}\right)^{-(1-\alpha) / 2} .
$$

Now, using Lemma 3.4 we obtain

$$
\begin{aligned}
\int_{t_{0}}^{t}\left\|e^{-(t-s) B} f(s)\right\|_{(n / \alpha, \infty)} & \leq c \int_{t_{0}}^{t}\left\|\nabla e^{-(t-s) B} f(s)\right\|_{(q, \infty)} \\
& \leq c\|f\|_{B C\left(\mathbb{R} ; L^{(l, \infty)}\right)}\left(t-t_{0}\right)^{-(1-\alpha) / 2+3 / 2-n / 2 l}
\end{aligned}
$$

for all $t_{0}<t<t_{0}+T$ with $c=c(n, q, l)$. Since $1 / l<1 / q+1 / n$, we have $(1-\alpha) / 2<3 / 2-n / 2 l$ and hence the above estimate yields

$$
\left(t-t_{0}\right)^{(1-\alpha) / 2}\left\|\int_{t_{0}}^{t} e^{-(t-s) B} f(s)\right\|_{(n / \alpha, \infty)} \leq c\|f\|_{B C\left(\mathbb{R}, L^{(l, \infty)}\right)} T^{(1-\alpha) / 2} .
$$

Consequently,

$$
\left\{\begin{aligned}
\sup _{t_{0}<t<t_{0}+T} & \left(t-t_{0}\right)^{(1-\alpha) / 2}\left\|w_{m+1}(t)\right\|_{(n / \alpha, \infty)} \\
& \leq K_{0,2}+c\|f\|_{B C\left(\mathbb{R} ; L^{(l, \infty)}\right)} T^{(1-\alpha) / 2}+C_{1,2} K_{m, 1} K_{m, 2}
\end{aligned}\right.
$$

Then, we can take $K_{m+1,1}, K_{m+1,2}$ being respectively,

$$
\begin{aligned}
& K_{m+1,1}=K_{0,1}+C_{1,1} K_{m, 1}^{2}+C_{2,1} K_{m, 2}, \\
& K_{m+1,2}=K_{0,2}+c\|f\|_{B C\left(\mathbb{R} ; L^{(l, \infty)}\right)} T^{(1-\alpha) / 2}+C_{1,2} K_{m, 1} K_{m, 2} .
\end{aligned}
$$

Setting $K_{m}=\max \left(K_{m, 1}, K_{m, 2}\right), m=1,2, \ldots$, from (3.43), (3.44), (3.45) and (3.46) we have

$$
\begin{gathered}
K_{m+1} \leq K_{0}+\widetilde{C} K_{m}^{2}+C_{2,1} K_{m}, \\
K_{0}=c_{1} T^{(1-\alpha) / 2} \max \left\{\|\mathbf{a}\|_{(n / \alpha, \infty)},\|b\|_{(n / \alpha, \infty)}+\|f\|_{B C\left(\mathbb{R} ; L^{(l, \infty)}\right)}\right\}
\end{gathered}
$$

and $\widetilde{C}=\max \left\{C_{1,1}, C_{1,2}\right\}$. If we consider

$$
C_{2,1}<1, K_{0}<\frac{\left(1-C_{2,1}\right)^{2}}{4 \widetilde{C}},
$$

we have that

$$
K_{m}<\frac{\left(1-C_{2,1}\right)-\sqrt{\left(1-C_{2,1}\right)^{2}-4 \widetilde{C} K_{0}}}{2 \widetilde{C}} \equiv k<\frac{1}{2 \widetilde{C}}, \forall m=0,1,2, \ldots
$$

Assuming (3.48) and working as Subsection 3.2, due to the uniform estimate with respect to $m$, we can conclude the existence of a couple $(\mathbf{v}, w)$ such that (3.40) holds and satisfying $(3.41)-(3.42)$. Thus, we finish the proof of Lemma 3.12. 
Lemma 3.13 If $K_{0}$ defined by (3.47) is small enough, then the limit $(\mathbf{v}, w)$ given by Lemma 3.12 satisfies the following estimate

$$
\left(t-t_{0}\right)^{1 / 2} \nabla(\mathbf{v}(\cdot), w(\cdot)) \in B C\left(\left(t_{0}, t_{0}+T\right) ; \mathbf{L}^{(n, \infty)}(\Omega) \times L^{(n, \infty)}(\Omega)\right)^{n},
$$

with

$$
\begin{aligned}
& \lim _{m \rightarrow \infty} \sup _{t_{0}<t<t_{0}+T}\left(t-t_{0}\right)^{1 / 2}\left\|\nabla \mathbf{v}_{m}(t)-\nabla \mathbf{v}(t)\right\|_{(n, \infty)}=0, \\
& \lim _{m \rightarrow \infty} \sup _{t_{0}<t<t_{0}+T}\left(t-t_{0}\right)^{1 / 2}\left\|\nabla w_{m}(t)-\nabla w(t)\right\|_{(n, \infty)}=0 .
\end{aligned}
$$

Proof. The proof is done by induction. In fact, we will prove that

$$
\begin{aligned}
& \sup _{t_{0}<t<t_{0}+T}\left(t-t_{0}\right)^{1 / 2}\left\|\nabla \mathbf{v}_{m}(t)\right\|_{(n, \infty)} \leq J_{m, 1}, \\
& \sup _{t_{0}<t<t_{0}+T}\left(t-t_{0}\right)^{1 / 2}\left\|\nabla w_{m}(t)\right\|_{(n, \infty)} \leq J_{m, 2},
\end{aligned}
$$

for some constants $J_{m, 1}, J_{m, 2}$ which are independent of $t_{0}, m=0,1, \ldots$

Note that by Lemma 3.2

$$
\left\|\nabla \mathbf{v}_{0}(t)\right\|_{(n, \infty)} \leq C\left(t-t_{0}\right)^{-1 / 2}\|\mathbf{a}\|_{(n, \infty)}, \quad\left\|\nabla w_{0}(t)\right\|_{(n, \infty)} \leq C\left(t-t_{0}\right)^{-1 / 2}\|b\|_{(n, \infty)},
$$

where $C=C(n)$ is independent of $t_{0}$. Hence we can take $J_{0,1}$ and $J_{0,2}$, being respectively, $C\|\mathbf{a}\|_{(n, \infty)}, C\|b\|_{(n, \infty)}$.

Supposed inequalities (3.51)-(3.52) are true. Then

$$
\begin{gathered}
\left\|\nabla \int_{t_{0}}^{t} e^{-(t-s) \mathbf{A}} P\left[\left(\mathbf{v}_{m} \cdot \nabla\right) \mathbf{v}_{m}\right]\right\|_{(n, \infty)} \leq \int_{t_{0}}^{t}(t-s)^{-n / 2(\alpha / n)-1 / 2}\left\|\mathbf{v}_{m}\right\|_{(n / \alpha, \infty)}\left\|\nabla \mathbf{v}_{m}\right\|_{(n, \infty)} \\
\quad \leq c K_{m, 1} J_{m, 1} \int_{t_{0}}^{t}(t-s)^{-\alpha / 2-1 / 2}\left(s-t_{0}\right)^{\alpha / 2-1} d s \\
\quad \leq c K_{m, 1} J_{m, 1}\left(t-t_{0}\right)^{-1 / 2} B((1-\alpha) / 2, \alpha / 2) \leq C_{3,1} k J_{m, 1}\left(t-t_{0}\right)^{-1 / 2},
\end{gathered}
$$

for all $t_{0}<t<t_{0}+T$, where $C_{3,1}=C_{3,1}(n, q)$ is independent of $t_{0}$. Moreover

$$
\begin{gathered}
\left\|\nabla \int_{t_{0}}^{t} e^{-(t-s) \mathbf{A}} P\left(\mathbf{g} w_{m}\right) d s\right\|_{(n, \infty)} \leq \int_{t_{0}}^{t}(t-s)^{-n / 2(\alpha / n)-1 / 2}\|\mathbf{g}\|_{(n, \infty)}\left\|w_{m}\right\|_{(n / \alpha, \infty)} d s \\
\quad \leq c\|\mathbf{g}\|_{(n, \infty)} K_{m, 2} \int_{t_{0}}^{t}(t-s)^{-(\alpha+1) / 2}\left(s-t_{0}\right)^{-(1-\alpha) / 2} d s \\
\quad \leq c B((1-\alpha) / 2,(1+\alpha) / 2) k\|\mathbf{g}\|_{(n, \infty)} \leq C_{4,1} k\|\mathbf{g}\|_{(n, \infty)} .
\end{gathered}
$$

Therefore,

$$
\sup _{t_{0}<t<t_{0}+T}\left(t-t_{0}\right)^{1 / 2}\left\|\nabla \mathbf{v}_{m+1}\right\|_{(n, \infty)} \leq J_{0,1}+C_{3,1} k J_{m, 1}+C_{4,1} k\|\mathbf{g}\|_{(n, \infty)} T^{1 / 2} .
$$

Now, for any $t, t_{0}<t<t_{0}+T$,

$$
\begin{gathered}
\left\|\nabla \int_{t_{0}}^{t} e^{-(t-s) B}\left(\mathbf{v}_{m} \cdot \nabla w_{m}\right) d s\right\|_{(n, \infty)} \leq \int_{t_{0}}^{t}(t-s)^{-n / 2(\alpha / n)-1 / 2}\left\|\mathbf{v}_{m}\right\|_{(n / \alpha, \infty)}\left\|\nabla w_{m}\right\|_{(n, \infty)} \\
\leq c K_{m, 1} J_{m, 2} \int_{t_{0}}^{t}(t-s)^{-\alpha / 2-1 / 2}\left(s-t_{0}\right)^{\alpha / 2-1} d s \leq C_{2,2} k J_{m, 2}\left(t-t_{0}\right)^{-1 / 2},
\end{gathered}
$$


where $C_{2,2}$ is independent of $t_{0}$. As

$$
\left\|\nabla \int_{t_{0}}^{t} e^{-(t-s) B} f(s) d s\right\|_{(n, \infty)} \leq c\left(t-t_{0}\right)^{-1 / 2}\|f\|_{B C\left(\mathbb{R} ; L^{(n, \infty)}\right)},
$$

we conclude that

$$
\sup _{t_{0}<t<t_{0}+T}\left(t-t_{0}\right)^{1 / 2}\left\|w_{m+1}(t)\right\|_{(n, \infty)} \leq J_{0,2}+C_{2,2} k J_{m, 2}+c\|f\|_{B C\left(\mathbb{R} ; L^{(n, \infty)}\right)} .
$$

Then we can take $J_{m+1,1}$ and $J_{m+1,2}$ being respectively,

$$
\begin{aligned}
& J_{m+1,1}=J_{0,1}+C_{3,1} k J_{m, 1}+C_{4,1} k\|\mathbf{g}\|_{(n, \infty)} T^{1 / 2}, \\
& J_{m+1,2}=J_{0,2}+C_{2,2} k J_{m, 2}+c\|f\|_{B C\left(\mathbb{R} ; L^{(n, \infty))} .\right.}
\end{aligned}
$$

Let $J_{m}=\max \left\{J_{m, 1}, J_{m, 2}\right\}, m=1,2, \ldots$ and

$$
J_{0}=\operatorname{Max}\left\{J_{0,1}+C_{4,1} k\|g\|_{(n, \infty)} T^{1 / 2}, J_{0,2}+c\|f\|_{B C\left(\mathbb{R} ; L^{(n, \infty)}\right)}\right\},
$$

then

$$
J_{m+1} \leq J_{0}+k \widehat{C} J_{m}
$$

where $\widehat{C}=\max \left\{C_{3,1}, C_{2,2}\right\}$. Consequently, if

$$
k<1 / \widehat{C}
$$

we have a uniform estimate for the sequence $\left\{J_{m}\right\}$ given by $J_{m} \leq \frac{J_{0}}{1-\widehat{C} k} \equiv J, m=0,1, \ldots$

Assuming (3.53), we can see that the limits $\mathbf{v}, w$ satisfy (3.50) and the proof of Lemma 3.13 is finished.

Lemma 3.14 The limit $(\mathbf{v}, w)$ given by Lemma 3.12 and Lemma 3.13 verifies

$$
\left(t-t_{0}\right)^{1 / 4}\left(\mathbf{v}(\cdot), w(\cdot) \in B C\left(\left(t_{0}, t_{0}+T\right) ; \mathbf{L}_{\sigma}^{2 n}(\Omega) \times L^{2 n}(\Omega)\right),\right.
$$

with

$$
\begin{aligned}
& \lim _{m \rightarrow \infty} \sup _{t_{0}<t<t_{0}+T}\left(t-t_{0}\right)^{1 / 4}\left\|\mathbf{v}_{m}(t)-\mathbf{v}(t)\right\|_{2 n}=0, \\
& \lim _{m \rightarrow \infty} \sup _{t_{0}<t<t_{0}+T}\left(t-t_{0}\right)^{1 / 4}\left\|w_{m}(t)-w(t)\right\|_{2 n}=0 .
\end{aligned}
$$

Proof. As the previous lemmas, the proof is done by induction. In fact, we will prove that there exist some constants $N_{m, 1}, N_{m, 2}$, which are independent of $t_{0}$, such that

$$
\left\|\mathbf{v}_{m}(t)\right\|_{2 n} \leq N_{m, 1}\left(t-t_{0}\right)^{-1 / 4}, \quad\left\|w_{m}(t)\right\|_{2 n} \leq N_{m, 2}\left(t-t_{0}\right)^{-1 / 4}
$$


Since $L^{\left(p_{0}, \infty\right)} \cap L^{\left(p_{1}, \infty\right)} \subset L^{p}$ and $\|f\|_{p} \leq C\left(p_{0}, p_{1}, \lambda\right)\|f\|_{\left(p_{0}, \infty\right)}^{1-\lambda}\|f\|_{\left(p_{1}, \infty\right)}^{\lambda}$ provided that $p_{0} \neq$ $p_{1}, 0<\lambda<1$ and $1 / p=(1-\lambda) / p_{0}+\lambda / p_{1}$, we have

$$
\left\|\mathbf{v}_{0}(t)\right\|_{2 n} \leq C\left(t-t_{0}\right)^{-1 / 4}\|\mathbf{a}\|_{(n, \infty)}, \quad\left\|w_{0}(t)\right\|_{2 n} \leq C\left(t-t_{0}\right)^{-1 / 4}\|b\|_{(n, \infty)},
$$

where $C=C(n)$ is independent of $t_{0}$. Hence, we define $N_{0,1}$ and $N_{0,2}$ as $C\|\mathbf{a}\|_{(n, \infty)}$ and $C\|b\|_{(n, \infty)}$, respectively.

Assuming true (3.54) for a given $m$, we can prove that (3.54) holds for the case $m+1$. In fact, note that for all $\phi \in \mathbf{C}_{0, \sigma}^{\infty}, \varphi \in C_{0}^{\infty}$, we get

$$
\begin{gathered}
\left|\left(-\int_{t_{0}}^{t} e^{-(t-s) \mathbf{A}} P\left[\left(\mathbf{v}_{m} \cdot \nabla\right) \mathbf{v}_{m}\right](s) d s, \boldsymbol{\phi}\right)\right| \leq \int_{t_{0}}^{t}\left\|\mathbf{v}_{m} \otimes \mathbf{v}_{m}\right\|_{n}\left\|\nabla e^{-(t-s) \mathbf{A}} \boldsymbol{\phi}\right\|_{n^{\prime}} d s \\
\leq C \int_{t_{0}}^{t}\left\|\mathbf{v}_{m}\right\|_{2 n}^{2}(t-s)^{-3 / 4}\|\boldsymbol{\phi}\|_{(2 n)^{\prime}} d s \leq c\left(t-t_{0}\right)^{-1 / 4} B(1 / 4,1 / 2) N_{m, 1}^{2}\|\boldsymbol{\phi}\|_{(2 n)^{\prime}} \\
\left|\left(-\int_{t_{0}}^{t} e^{-(t-s) B}\left(\mathbf{v}_{m} \cdot \nabla w_{m}\right)(s) d s, \varphi\right)\right| \leq \int_{t_{0}}^{t}\left\|w_{m} \cdot \mathbf{v}_{m}\right\|_{n}\left\|\nabla e^{-(t-s) B} \varphi\right\|_{n^{\prime}} d s \\
\leq C \int_{t_{0}}^{t}\left\|\mathbf{v}_{m}\right\|_{2 n}\left\|w_{m}\right\|_{2 n}(t-s)^{-3 / 4}\|\varphi\|_{(2 n)^{\prime}} d s \leq c\left(t-t_{0}\right)^{-1 / 4} B(1 / 4,1 / 2) N_{m, 1} N_{m, 2}\|\varphi\|_{(2 n)^{\prime}} .
\end{gathered}
$$

Hence by duality

$$
\begin{aligned}
& \| \int_{t_{0}}^{t} e^{-(t-s) \mathbf{A} P\left(\mathbf{v}_{m} \cdot \nabla \mathbf{v}_{m}\right)(s) d s \|_{2 n}} \leq C_{1,1} N_{m, 1}^{2}\left(t-t_{0}\right)^{-1 / 4} \\
& \left\|\int_{t_{0}}^{t} e^{-(t-s) B}\left(\mathbf{v}_{m} \cdot \nabla w_{m}\right)(s) d s\right\|_{2 n} \leq C_{1,2} N_{m, 1} N_{m, 2}\left(t-t_{0}\right)^{-1 / 4} .
\end{aligned}
$$

We also note that

$$
\begin{gathered}
\left\|\int_{t_{0}}^{t} e^{-(t-s) \mathbf{A}} P\left(\mathbf{g} w_{m}\right)(s) d s\right\|_{2 n} \leq \int_{t_{0}}^{t}(t-s)^{-1 / 2}\|\mathbf{g}\|_{(n, \infty)}\left\|w_{m}(s)\right\|_{(2 n)} d s \\
\leq c\|\mathbf{g}\|_{(n, \infty)} B(1 / 2,3 / 4)\left(t-t_{0}\right)^{1 / 4} \leq C_{2,1} N_{m, 2}\left(t-t_{0}\right)^{1 / 4}
\end{gathered}
$$

and

$$
\left\|\int_{t_{0}}^{t} e^{-(t-s) B} f(s) d s\right\|_{2 n} \leq \int_{t_{0}}^{t}(t-s)^{-1 / 4}\|f(s)\|_{(n, \infty)} d s \leq c\|f\|_{B C\left(\mathbb{R} ; L^{(n, \infty)}\right)}\left(t-t_{0}\right)^{3 / 4} .
$$

The inequalities above imply that

$$
\begin{aligned}
\sup _{t_{0}<t<t_{0}+T}\left(t-t_{0}\right)^{1 / 4}\left\|\mathbf{v}_{m+1}\right\|_{2 n} & \leq N_{0,1}+C_{1,1} N_{m, 1}^{2}+C_{2,1} N_{m, 2} T^{1 / 2} \\
\sup _{t_{0}<t<t_{0}+T}\left(t-t_{0}\right)^{1 / 4}\left\|w_{m+1}\right\|_{2 n} & \leq N_{0,2}+C_{1,2} N_{m, 1} N_{m, 2}+c\|f\|_{B C\left(\mathbb{R} ; L^{(n, \infty)}\right)} T .
\end{aligned}
$$

As before, setting $N_{m}=\max \left(N_{m, 1}, N_{m, 2}\right), m=1,2, \ldots$ and $N_{0}=\max \left(N_{0,2}+c\|f\|_{B C\left(\mathbb{R} ; L^{(n, \infty)}\right)} T, N_{0,1}\right)$, we obtain $N_{m+1} \leq N_{0}+\widetilde{C} N_{m}^{2}+\widetilde{C}_{2,1} N_{m}$, with $\widetilde{C}_{2,1}=C_{2,1} T^{1 / 2}$ where $\widetilde{C}=\max \left(C_{1,1}, C_{1,2}\right)$. If we consider

$$
C_{2,1}<1, \quad N_{0}<\frac{\left(1-C_{2,1}\right)^{2}}{4 \widetilde{C}}
$$


we have that the sequence $\left\{N_{m}\right\}_{m=0}^{m=\infty}$ is bounded with

$$
N_{m} \leq \frac{\left(1-C_{2,1}\right)-\sqrt{\left(1-C_{2,1}\right)^{2}-4 N_{0} \widetilde{C}}}{2 \widetilde{C}}, m=0,1, \ldots
$$

Assuming (3.55) and working as Lemma 3.12 and Lemma 3.13, we conclude the proof.

Lemma 3.15 The limit $(\mathbf{v}, w)$ given by Lemma 3.12 and Lemma 3.13 verifies

$$
(\mathbf{v}, w) \in B C\left(\left(t_{0}, t_{0}+T\right) ; \mathbf{L}_{\sigma}^{(n, \infty)}(\Omega) \cap \mathbf{L}_{\sigma}^{\left(q^{*}, \infty\right)}(\Omega) \times L^{(n, \infty)}(\Omega) \cap L^{\left(q^{*}, \infty\right)}(\Omega)\right),
$$

with

$$
\begin{aligned}
& \sup _{t_{0}<t<t_{0}+T}\left\|\mathbf{v}_{m}(t)\right\|_{(n / s, \infty)} \leq M_{1, s, m}, \quad s=\alpha, \quad s=1, \\
& \sup _{t_{0}<t<t_{0}+T}\left\|w_{m}(t)\right\|_{(n / s, \infty)} \leq M_{2, s, m}, \quad s=\alpha, \quad s=1,
\end{aligned}
$$

and $M_{1, s, m}, M_{2, s, m}$ independent of $t_{0}$.

Proof. Computations similar to Lemma 3.12, Lemma 3.13, yield to

$$
\begin{array}{ll}
M_{1, \alpha, 0}=C\|\mathbf{a}\|_{(n / \alpha, \infty)}, & M_{1,1,0}=C\|\mathbf{a}\|_{(n, \infty)}, \\
M_{2, \alpha, 0}=C\|b\|_{(n / \alpha, \infty)}, & M_{2,1,0}=C\|b\|_{(n, \infty)},
\end{array}
$$

where $C=C(n, s)$ is independent of $t_{0}$. Suppose by induction that (3.57), (3.58) are true. Note that

$$
\begin{aligned}
\left|\left(-\int_{t_{0}}^{t} e^{-(t-s) \mathbf{A}} P\left(\mathbf{v}_{m} \cdot \nabla \mathbf{v}_{m}\right)(s) d s, \boldsymbol{\phi}\right)\right| \leq\left|\int_{t_{0}}^{t}\left(\mathbf{v}_{m} \otimes \mathbf{v}_{m}(s), \nabla e^{-(t-s) \mathbf{A}} \boldsymbol{\phi}\right) d s\right| \\
\quad \leq \int_{t_{0}}^{t}\left\|\mathbf{v}_{m}\right\|_{(n / \alpha, \infty)}\left\|\mathbf{v}_{m}\right\|_{(n / s, \infty)}\left\|\nabla e^{-(t-s) \mathbf{A}} \boldsymbol{\phi}\right\|_{(n /(n-\alpha-s), 1)} d s \\
\quad \leq C K_{m, 1} M_{1, s, m} \int_{t_{0}}^{t}(t-s)^{-\alpha / 2-1 / 2}\left(s-t_{0}\right)^{-(1-\alpha) / 2} \cdot\|\phi\|_{(n /(n-s), 1)} d s \\
\quad \leq C k M_{1, s, m} B((1-\alpha) / 2,(1+\alpha) / 2)\|\phi\|_{(n /(n-s), 1)},
\end{aligned}
$$

for all $\phi \in \mathbf{C}_{0, \sigma}^{\infty}$ and all $t_{0}<t<t_{0}+T$ and $C=C(n, q, s)$ independent of $t_{0}$. Consequently, by duality, for $s=1, \alpha$, we have

$$
\sup _{t_{0}<t<t_{0}+T}\left\|\int_{t_{0}}^{t} e^{-(t-s) \mathbf{A}} P\left(\mathbf{v}_{m} \cdot \nabla \mathbf{v}_{m}\right)(s) d s\right\|_{(n / s, \infty)} \leq C_{5,1} k M_{1, s, m},
$$

where $C_{5,1}$ independent of $t_{0}$. Note that

$$
\begin{aligned}
\| \int_{t_{0}}^{t} e^{-(t-s) \mathbf{A}} P\left(\mathbf{g} w_{m}\right)(s) d s & \left\|_{(n / s, \infty)} \leq c\right\| \mathbf{g}\left\|_{(b, \infty)} \int_{t_{0}}^{t}(t-s)^{-n / 2 b}\right\| w_{m}(s) \|_{(n / s, \infty)} d s \\
& \leq c\|\mathbf{g}\|_{(b, \infty)}\left(t-t_{0}\right)^{1-n / 2 b} \leq C_{6,1} M_{2, s, m}\left(t-t_{0}\right)^{1-n / 2 b}
\end{aligned}
$$




$$
\left\|\int_{t_{0}}^{t} e^{-(t-s) B} f(s) d s\right\|_{(n / \alpha, \infty)} \leq c\|f\|_{B C\left(\mathbb{R} ; L^{(l, \infty)}\right)} T^{a}
$$

with $a=\frac{\alpha}{2}-\frac{n}{2 l}+1>0$. Moreover, for all $\varphi \in C_{0}^{\infty}$ and all $t_{0}<t<t_{0}+T$,

$$
\begin{aligned}
\mid(- & \left.\left.\int_{t_{0}}^{t} e^{-(t-s) B}\left(\mathbf{v}_{m} \cdot \nabla w_{m}\right)(s) d s, \varphi\right)|\leq| \int_{t_{0}}^{t}\left(w_{m} \cdot \mathbf{v}_{m}(s), \nabla e^{-(t-s) B}\right) \varphi\right) d s \mid \\
& \leq \int_{t_{0}}^{t}\left\|\mathbf{v}_{m}\right\|_{(n / \alpha, \infty)}\left\|w_{m}\right\|_{(n / s, \infty)}\left\|\nabla e^{-(t-s) B} \varphi\right\|_{(n /(n-\alpha-s), 1)} d s \\
& \leq c K_{m, 1} M_{2, s, m}\left(\int_{t_{0}}^{t}(t-s)^{-\alpha / 2-1 / 2}\left(s-t_{0}\right)^{-(1-\alpha) / 2} d s\right)\|\varphi\|_{(n /(n-s), 1)} \\
& \leq c k M_{2, s, m} B((1-\alpha) / 2,(1+\alpha) / 2)\|\varphi\|_{(n /(n-s), 1)} .
\end{aligned}
$$

Thus, for $s=1, \alpha$,

$$
\sup _{t_{0}<t<t_{0}+T}\left\|\int_{t_{0}}^{t} e^{-(t-s) B}\left(\mathbf{v}_{m} \cdot \nabla w_{m}\right)(s) d s\right\|_{(n / s, \infty)} \leq C_{4,2} k M_{2, s, m},
$$

where $C_{4,2}$ is independent of $t_{0}$. Hence, from (3.59)-(3.60) we can take

$$
\begin{aligned}
& M_{1, s, m+1}=M_{1, s, 0}+C_{5,1} k M_{1, s, m}+C_{6,1} M_{2, s, m}, \\
& M_{2, s, m+1}=M_{2, s, 0}+c\|f\|_{B C\left(\mathbb{R} ; L^{(l, \infty)}\right)} T^{a}+C_{4,2} k M_{2, s, m} .
\end{aligned}
$$

Setting

$$
\begin{aligned}
M_{s, m} & =\max \left\{M_{1, s, m}, M_{2, s, m}\right\}, \\
M_{s, 0} & =\max \left\{M_{1, s, 0}, M_{2, s, 0}+c\|f\|_{B C\left(\mathbb{R} ; L^{(l, \infty)}\right)} T^{a}\right\}, \\
\breve{C} & =\max \left\{C_{5,1}, C_{4,2}\right\},
\end{aligned}
$$

from (3.61),(3.62) we obtain $M_{s, m+1} \leq M_{s, 0}+k \breve{C} M_{s, m}+C_{6,1} M_{s, m}$, for $m=0,1, \ldots, s=1, \alpha$. Then, if

$$
k \breve{C}+C_{6,1}<1
$$

we have $M_{s, m} \leq \frac{M_{s, 0}}{1-k \breve{C}-C_{6,1}}, m=0,1, \ldots, s=1, \alpha$, which yields to (3.56) with (3.57)-(3.58).

Now, we continue the proof of Proposition 3.11. We can see that under conditions (3.48), (3.53) and (3.63), the limit $(\mathbf{v}, w)$ belongs to the class required in Proposition 3.11. Moreover, then following convergences hold in $\mathbf{L}_{\sigma}^{(n, \infty)}(\Omega), \mathbf{L}_{\sigma}^{(n, \infty)}(\Omega)$ and $\mathbf{L}^{(n, \infty)}(\Omega)$, respectively

$$
\begin{array}{r}
\int_{t_{0}}^{t} e^{-(t-s) \mathbf{A}} P\left[\left(\mathbf{v}_{m} \cdot \nabla\right) \mathbf{v}_{m}\right](s) d s \longrightarrow \int_{t_{0}}^{t} e^{-(t-s) \mathbf{A}} P[(\mathbf{v} \cdot \nabla) \mathbf{v}](s) d s \\
\int_{t_{0}}^{t} e^{-(t-s) \mathbf{A}} P\left(w_{m} \mathbf{g}\right)(s) d s \longrightarrow \int_{t_{0}}^{t} e^{-(t-s) \mathbf{A}} P(w \mathbf{g})(s) d s \\
\int_{t_{0}}^{t} e^{-(t-s) B} P\left[\left(\mathbf{v}_{m} \cdot \nabla\right) w_{m}\right](s) d s \longrightarrow \int_{t_{0}}^{t} e^{-(t-s) \mathbf{A}} P[(\mathbf{v} \cdot \nabla) w](s) d s
\end{array}
$$


uniformly in $t \in\left(t_{0}, t_{0}+t\right)$ as $m \rightarrow \infty$. In fact, note that by Lemma 3.2, Lemma 3.12 and Lemma 3.13, we have

$$
\begin{aligned}
& \left\|\int_{t_{0}}^{t} e^{-(t-s) \mathbf{A}} P\left[\left(\mathbf{v}_{m} \cdot \nabla\right) \mathbf{v}_{m}\right](s) d s-\int_{t_{0}}^{t} e^{-(t-s) \mathbf{A}} P[(\mathbf{v} \cdot \nabla) \mathbf{v}](s) d s\right\|_{(n, \infty)} \\
& \quad \leq \int_{t_{0}}^{t}(t-s)^{-\alpha / 2}\left\|\mathbf{v}_{m}(s)-\mathbf{v}(s)\right\|_{(n / \alpha, \infty)}\left\|\nabla \mathbf{v}_{m}(s)\right\|_{(n, \infty)} d s \\
& \quad+\int_{t_{0}}^{t}(t-s)^{-\alpha / 2}\|\mathbf{v}(s)\|_{(n / \alpha, \infty)}\left\|\nabla\left(\mathbf{v}_{m}(s)-\mathbf{v}(s)\right)\right\|_{(n, \infty)} d s \\
& \quad \leq c B(1-\alpha / 2,1 / 2) J \sup _{t_{0}<s<t_{0}+T}\left(s-t_{0}\right)^{(1-\alpha) / 2}\left\|\mathbf{v}_{m}(s)-\mathbf{v}(s)\right\|_{(n / \alpha, \infty)} \\
& \left.\quad+B(1-\alpha / 2,(\alpha+1) / 2) k \sup _{t_{0}<s<t_{0}+T}\left(s-t_{0}\right)^{1 / 2}\left\|\nabla \mathbf{v}_{m}(s)-\nabla \mathbf{v}(s)\right\|_{(n, \infty)}\right),
\end{aligned}
$$

which converges to 0 . On the other hand

$$
\begin{aligned}
& \left\|\int_{t_{0}}^{t} e^{-(t-s) \mathbf{A}} P\left(w_{m} g\right)(s) d s-\int_{t_{0}}^{t} e^{-(t-s) \mathbf{A}} P(w \mathbf{g})(s) d s\right\|_{(n, \infty)} \\
& \leq \int_{t_{0}}^{t}\left\|e^{-(t-s) \mathbf{A}} P\left(\left(w_{m}-w\right) \mathbf{g}\right)(s)\right\|_{(n, \infty)} d s \\
& \leq \int_{t_{0}}^{t}(t-s)^{-n / 2 b+(1-\alpha) / 2}\|\mathbf{g}\|_{(b, \infty)}\left\|w_{m}(s)-w(s)\right\|_{(n / \alpha, \infty)} \\
& \leq c T^{1-n / 2 b} \sup _{t_{0}<s<t_{0}+T}\left(s-t_{0}\right)^{(1-\alpha) / 2}\left\|w_{m}(s)-w(s)\right\|_{(n / \alpha, \infty)} \longrightarrow 0 .
\end{aligned}
$$

Analogously we obtain (3.64). Now we will prove the weak continuity on the initial dada. Firstly, we note that for any $\phi \in \mathbf{L}_{\sigma}^{\left(n^{\prime}, 1\right)}(\Omega)$ and $\varphi \in L^{\left(n^{\prime}, 1\right)}(\Omega)$ we have

$$
\begin{aligned}
\left|\left(e^{-\left(t-t_{0}\right) \mathbf{A}} \mathbf{a}-\mathbf{a}, \boldsymbol{\phi}\right)\right| & =\left|\left(\mathbf{a}, e^{-\left(t-t_{0}\right) \mathbf{A}} \boldsymbol{\phi}-\boldsymbol{\phi}\right)\right| \\
& \leq\|\mathbf{a}\|_{(n, \infty)}\left\|e^{-\left(t-t_{0}\right) \mathbf{A}} \boldsymbol{\phi}-\boldsymbol{\phi}\right\|_{\left(n^{\prime}, 1\right)} \rightarrow 0, \quad t \rightarrow t_{0}^{+} . \\
\left|\left(e^{-\left(t-t_{0}\right) B} b-b, \varphi\right)\right| & =\mid\left(b, e^{\left.-\left(t-t_{0}\right) \mathbf{A} \varphi-\varphi\right) \mid}\right. \\
& \leq\|b\|_{(n, \infty)}\left\|e^{-\left(t-t_{0}\right) B} \varphi-\varphi\right\|_{\left(n^{\prime}, 1\right)} \rightarrow 0, \quad t \rightarrow t_{0}^{+} .
\end{aligned}
$$

As $\|\mathbf{v}\|_{\left(q^{*}, \infty\right)},\|w\|_{\left(q^{*}, \infty\right)} \leq c$ and $\left(t-t_{0}\right)^{1 / 4}\|\mathbf{v}\|_{2 n},\left(t-t_{0}\right)^{1 / 4}\|w\|_{2 n} \leq c$, we have

$$
\begin{aligned}
& \lim _{t \rightarrow t_{0}}\left(\int_{t_{0}}^{t} e^{-(t-s) \mathbf{A}} P(w \mathbf{g})(s) d s-\int_{t_{0}}^{t} e^{-(t-s) \mathbf{A}} P[(\mathbf{v} \cdot \nabla) \mathbf{v}](s) d s, \boldsymbol{\phi}\right)=0, \\
& \lim _{t \rightarrow t_{0}}\left(\int_{t_{0}}^{t} e^{-(t-s) B} f(s) d s-\int_{t_{0}}^{t} e^{-(t-s) B}(\mathbf{v} \cdot \nabla w)(s) d s, \varphi\right)=0
\end{aligned}
$$

Indeed, note that when $t \rightarrow t_{0}^{+}$,

$$
\begin{aligned}
\int_{t_{0}}^{t}\left(e^{-(t-s) B}\right. & {[(\mathbf{v} \cdot \nabla) w], \varphi) \leq \int_{t_{0}}^{t}\|\mathbf{v}\|_{\left(q^{*}, \infty\right)}\|w\|_{2 n}\left\|\nabla e^{-(t-s) B} \varphi\right\|_{\left(1-1 / q^{*}-1 / 2 n, 1\right)} } \\
& \leq c \int_{t_{0}}^{t}\left(s-t_{0}\right)^{-1 / 4}(t-s)^{-n / 2 q+1 / 4}\|\varphi\|_{\left(n^{\prime}, 1\right)} d s \\
& \leq c\left(t-t_{0}\right)^{1-n / 2 q} B(3 / 4,-n / 2 q+5 / 4)\|\varphi\|_{\left(n^{\prime}, 1\right)} \rightarrow 0,
\end{aligned}
$$




$$
\begin{gathered}
\int_{t_{0}}^{t}\left(e^{-(t-s) \mathbf{A}} P[(\mathbf{v} \cdot \nabla) \mathbf{v}], \boldsymbol{\phi}\right) \leq \int_{t_{0}}^{t}\|\mathbf{v}\|_{\left(q^{*}, \infty\right)}\|\mathbf{v}\|_{2 n}\left\|\nabla e^{-(t-s) \mathbf{A}} \boldsymbol{\phi}\right\|_{\left(1-1 / q^{*}-1 / 2 n, 1\right)} \\
\leq c \int_{t_{0}}^{t}\left(s-t_{0}\right)^{-1 / 4}(t-s)^{-n / 2 q+1 / 4}\|\boldsymbol{\phi}\|_{\left(n^{\prime}, 1\right)} d s \\
\leq c\left(t-t_{0}\right)^{1-n / 2 q} B(3 / 4,-n / 2 q+5 / 4)\|\boldsymbol{\phi}\|_{\left(n^{\prime}, 1\right)} \rightarrow 0 \\
\int_{t_{0}}^{t}\left(e^{-(t-s) \mathbf{A}} P(w \mathbf{g}), \boldsymbol{\phi}\right) d s \leq \int_{t_{0}}^{t}\left\|e^{-(t-s) \mathbf{A}} P(w \mathbf{g})\right\|\left\|_{(n, \infty)}\right\| \boldsymbol{\phi} \|_{\left(n^{\prime}, 1\right)} d s \\
\leq c\|w\|_{q^{*}}\|\mathbf{g}\|_{(n, \infty)}\|\boldsymbol{\phi}\|_{\left(n^{\prime}, 1\right)} \int_{t_{0}}^{t}\left(s-t_{0}\right)^{-n / 2 q^{*}} d s \\
\leq c\left(t-t_{0}\right)^{1-n / 2 q^{*}}\|w\|_{q^{*}}\|\mathbf{g}\|_{(n, \infty)}\|\boldsymbol{\phi}\|_{\left(n^{\prime}, 1\right)} \rightarrow 0
\end{gathered}
$$

and

$$
\begin{aligned}
\int_{t_{0}}^{t}\left(e^{-(t-s) B} f(s), \varphi\right) d s & \leq \int_{t_{0}}^{t}\left\|e^{-(t-s) B} f(s)\right\|_{(n, \infty)}\|\varphi\|_{\left(n^{\prime}, 1\right)} d s \\
& \leq c \int_{t_{0}}^{t}\|f\|_{(n, \infty)} d s\|\varphi\|_{\left(n^{\prime}, 1\right)} \leq c\left(t-t_{0}\right) \rightarrow 0 .
\end{aligned}
$$

Collecting all the previous convergences and letting $m \rightarrow \infty$ in (3.36)-(3.37), we see that $(\mathbf{v}, w)$ is a solution of (3.34)-(3.35). Finally, we will estimate the time-interval $T$ of existence in terms of the prescribed data. As $k$ is determined by (3.49), there exists a constant $\tilde{k}$ independent of $t_{0}$ such that if $K_{0} \leq \tilde{k}$, then conditions (3.48),(3.53), (3.63) are satisfied. Now, from (3.47) we see that $T$ may be chosen as (3.33).

Remark 3.16 The solution (v, w) of integral equations (3.34)-(3.35) satisfies $(\mathbf{v}, w) \in B C\left(t_{0}, t_{0}+\right.$ $\left.T ; \mathbf{L}_{\sigma}^{p}(\Omega) \times L^{p}(\Omega)\right)$, for all $p \in\left(n, q^{*}\right)$, with $\|\mathbf{v}\|_{p} \leq C\|\mathbf{v}\|_{(n, \infty)}^{1-\lambda}\|\mathbf{u}\|_{\left(q^{*}, \infty\right)}^{\lambda},\|w\|_{p} \leq C\|w\|_{(n, \infty)}^{1-\lambda}\|w\|_{\left(q^{*}, \infty\right)}^{\lambda}$, where $\lambda$ is such that $1 / p=(1-\lambda) / n+\lambda / q^{*}$.

Note that being a and $b$ elements of $\mathbf{L}_{\sigma}^{(n, \infty)}(\Omega) \cap \mathbf{L}_{\sigma}^{\left(q^{*}, \infty\right)}(\Omega)$ and $L^{(n, \infty)}(\Omega) \cap L^{\left(q^{*}, \infty\right)}(\Omega)$, respectively, we have that a and $b$ belong to space $\mathbf{L}_{\sigma}^{r^{*}}(\Omega)$ and $L^{r^{*}}(\Omega)$, respectively. Consequently, the norms $\left\|e^{-\left(t-t_{0}\right) \mathbf{A}} \mathbf{a}\right\|_{r^{*}} \leq C\|\mathbf{a}\|_{r^{*}}$ and $\left\|e^{-\left(t-t_{0}\right) B} b\right\|_{r^{*}} \leq C\|b\|_{r^{*}}$ are finite. Moreover, it is not difficult to see that

$$
\begin{gathered}
\left|\left(\int_{t_{0}}^{t} e^{-(t-s) \mathbf{A}} P[(\mathbf{v} \cdot \nabla) \mathbf{v}], \boldsymbol{\phi}\right)\right| \leq C\left(\int_{t_{0}}^{t}\|\mathbf{v}\|_{r^{*}}^{2}(t-s)^{-\frac{n}{2}\left(\frac{1}{\left(r^{*}\right)^{\prime}}-\frac{1}{\left(r^{*} / 2\right)^{\prime}}\right)-\frac{1}{2}}\right)\|\boldsymbol{\phi}\|_{\left(r^{*}\right)^{\prime}} \\
\leq C\|\mathbf{v}\|_{r^{*}}^{2}\left(\int_{t_{0}}^{t}(t-s)^{-\frac{n}{2 r^{*}}-\frac{1}{2}} d s\right)\|\boldsymbol{\phi}\|_{\left(r^{*}\right)^{\prime}} \leq C\|\boldsymbol{\phi}\|_{\left(r^{*}\right)^{\prime}}
\end{gathered}
$$

for all $\phi \in \mathbf{C}_{0, \sigma}^{\infty}$. By duality, we have that $\int_{t_{0}}^{t} e^{-(t-s) \mathbf{A}} P[(\mathbf{v} \cdot \nabla) \mathbf{v}](s) d s \in \mathbf{L}_{\sigma}^{r^{*}}(\Omega)$.

Analogously, we can see that

$$
\int_{t_{0}}^{t} e^{-(t-s) B}(\mathbf{v} \cdot \nabla w)(s) d s+\int_{t_{0}}^{t} e^{-(t-s) \mathbf{A}} P(w \mathbf{g})(s) d s \in \mathbf{L}^{r^{*}}(\Omega), \int_{t_{0}}^{t} e^{-(t-s) B} f(s) d s \in L^{r^{*}}(\Omega) .
$$


Therefore we conclude that equalities (3.34)-(3.35) are satisfied in $\mathbf{L}_{\sigma}^{r^{*}}(\Omega)$ and $L^{r^{*}}(\Omega)$, respectively. The proof of Proposition 3.11 is finished.

Proof of Theorem 3.10. By the hypothesis of Theorem 3.10, we can apply Proposition 3.11 and hence, there exist $T \in(0,1]$ and functions $\mathbf{v}$ and $w$ satisfying:

$$
\left\{\begin{array}{l}
\mathbf{v} \in B C_{w}\left(\left[t_{0}, t_{0}+T\right) ; \mathbf{L}_{\sigma}^{(n, \infty)}(\Omega)\right) \cap B C\left(\left[t_{0}, t_{0}+T\right) ; \mathbf{L}_{\sigma}^{\left(q^{*}, \infty\right)}(\Omega)\right), \\
w \in B C_{w}\left(\left[t_{0}, t_{0}+T\right) ; L^{(n, \infty)}(\Omega)\right) \cap B C\left(\left[t_{0}, t_{0}+T\right) ; L^{\left(q^{*}, \infty\right)}(\Omega)\right), \\
\left(t-t_{0}\right)^{1 / 2} \nabla \mathbf{v} \in B C\left(\left(t_{0}, t_{0}+T\right) ; \mathbf{L}^{(n, \infty)}(\Omega)\right)^{n} \\
\left(t-t_{0}\right)^{1 / 2} \nabla w \in B C\left(\left(t_{0}, t_{0}+T\right) ; L^{(n, \infty)}(\Omega)\right)^{n}
\end{array}\right.
$$

in such a way that for all $t_{0} \in \mathbb{R}$ the integral system (3.34)-(3.35) is satisfied in the $L^{r^{*}}$-norm.

Firstly, we study the uniqueness of that solution. Let $\left(\mathbf{v}_{1}, w_{1}\right)$ another solution of (3.34)(3.35), in the class given by Proposition 3.11, with the same initial condition. Let $(\mathbf{V}, W)=$ $\left(\mathbf{v}-\mathbf{v}_{1}, w-w_{1}\right)$. Note that $(\mathbf{V}(t), W(t)) \in \mathbf{L}_{\sigma}^{n}(\Omega) \times L^{n}(\Omega)$ for all $0<t<T$, with

$$
\sup _{t_{0}<t<T}\|\mathbf{V}(t)\|_{n}<\infty, \quad \sup _{t_{0}<t<T}\|W(t)\|_{n}<\infty .
$$

In fact, taking $n<q<\min \left\{2 n, r^{*}\right\}$ we have for all $\phi \in \mathbf{C}_{0, \sigma}^{\infty}$ and all $t_{0}<t<T$,

$$
\begin{aligned}
& |(\mathbf{V}(t), \boldsymbol{\phi})| \leq\left|\int_{t_{0}}^{t}\left(\mathbf{v}-\mathbf{v}_{1} \otimes \mathbf{v}_{1}, \nabla e^{-(t-s) \mathbf{A}} \boldsymbol{\phi}\right) d s\right|+\left|\int_{t_{0}}^{t}\left(e^{-(t-s) \mathbf{A}} P(W \mathbf{g}), \boldsymbol{\phi}\right) d s\right| \\
& \quad \leq C(n, q)\left\{\left(\sup _{t_{0}<s<T} s^{n / 2(1 / n-1 / q)}\|\mathbf{v}\|_{q}\right)^{2}+\left(\sup _{t_{0}<s<T} s^{n / 2(1 / n-1 / q)}\left\|\mathbf{v}_{1}\right\|_{q}\right)^{2}\right\}\|\boldsymbol{\phi}\|_{n^{\prime}} \\
& \quad+C(n, q)\left\{\left(\sup _{t_{0}<s<T} s^{n / 2(1 / n-1 / q)}\|w(s)\|_{q}+\sup _{t_{0}<s<T} s^{n / 2(1 / n-1 / q)}\left\|w_{1}(s)\right\|_{q}\right)\right. \\
& \left.\quad \times\left(\sup _{t_{0}<s<T} s^{1 / 2}\|\mathbf{g}\|_{(n, \infty)}\right)\right\}\|\boldsymbol{\phi}\|_{n^{\prime}} .
\end{aligned}
$$

Analogously, for all $\varphi \in C_{0}^{\infty}$ and all $t_{0}<t<T$

$$
\begin{aligned}
|(W(t), \varphi)| & \leq C(n, q)\left\{\sup _{t_{0}<s<T} s^{n / 2(1 / n-1 / q)}\|\mathbf{v}(s)\|_{q} \sup _{t_{0}<s<T} s^{n / 2(1 / n-1 / q)}\|w(s)\|_{q}\right. \\
& \left.+\sup _{t_{0}<s<T} s^{n / 2(1 / n-1 / q)}\left\|\mathbf{v}_{1}(s)\right\|_{q} \sup _{t_{0}<s<T} s^{n / 2(1 / n-1 / q)}\left\|w_{1}(s)\right\|_{q}\right\}\|\varphi\|_{n^{\prime}} .
\end{aligned}
$$


By duality we can conclude (3.65). We define $K_{\mathbf{V}}(t) \equiv \sup _{t_{0}<t<T}\|\mathbf{V}(t)\|_{(n, \infty)}$ and $K_{W}(t) \equiv$ $\sup _{t_{0}<t<T}\|W(t)\|_{(n, \infty)}$. Let $p$ satisfying $1 / p=1-1 / n-1 / r^{*}$. Then

$$
\begin{aligned}
|(\mathbf{V}(t), \boldsymbol{\phi})| & \leq\left|\int_{t_{0}}^{t}\left(\mathbf{V} \otimes \mathbf{v}(s)-\mathbf{v}_{1}, \nabla e^{-(t-s) \mathbf{A}} \boldsymbol{\phi}\right) d s\right|+\left|\int_{t_{0}}^{t}\left(e^{-(t-s) \mathbf{A}} P(W \mathbf{g})(s), \boldsymbol{\phi}\right) d s\right| \\
& \leq \int_{t_{0}}^{t}\left(\|\mathbf{v}(s)\|_{r^{*}}+\left\|\mathbf{v}_{1}(s)\right\|_{r^{*}}\right)\|\mathbf{V}(s)\|_{n}\left\|\nabla e^{-(t-s) L} \boldsymbol{\phi}\right\|_{p} d s \\
& +\int_{t_{0}}^{t}(t-s)^{-1 / 2}\|W(s)\|_{n}\|\mathbf{g}\|_{(n, \infty)}\|\boldsymbol{\phi}\|_{n^{\prime}} d s \\
& \leq C K_{\mathbf{V}}(t)\left(\sup _{t_{0}<s<t_{0}+t}\left(s-t_{0}\right)^{n / 2\left(1 / n-1 / r^{*}\right)}\|\mathbf{v}(s)\|_{r^{*}}\right. \\
& \left.+\sup _{t_{0}<s<t_{0}+t}\left(s-t_{0}\right)^{n / 2\left(1 / n-1 / r^{*}\right)}\left\|\mathbf{v}_{1}(s)\right\|_{r^{*}}\right) \\
& \times\left(\int_{t_{0}}^{t}(t-s)^{-n / 2\left(1 / n^{\prime}-1 / p\right)-1 / 2}\left(s-t_{0}\right)^{-n / 2\left(1 / n-1 / r^{*}\right)} d s\right)\|\boldsymbol{\phi}\|_{n^{\prime}} \\
& +K_{W} \sup _{t_{0}<s<t_{0}+t}\left(s-t_{0}\right)^{1 / 2}\|\mathbf{g}\|_{(n, \infty)}\left(\int_{t_{0}}^{t}(t-s)^{-1 / 2}\left(s-t_{0}\right)^{-1 / 2} d s\right)\|\boldsymbol{\phi}\|_{n^{\prime}}
\end{aligned}
$$

for all $\phi \in \mathbf{C}_{0, \sigma}^{\infty}$ and for all $t_{0}<t<T$. Then, using duality we obtain

$$
\begin{gathered}
\|\mathbf{V}(t)\|_{n} \leq K_{\mathbf{V}}(t)\left(\sup _{t_{0}<s<t_{0}+t}\left(s-t_{0}\right)^{n / 2\left(1 / n-1 / r^{*}\right)}\|\mathbf{v}(s)\|_{r^{*}+}\right. \\
\left.+\sup _{t_{0}<s<t_{0}+t}\left(s-t_{0}\right)^{n / 2\left(1 / n-1 / r^{*}\right)}\left\|\mathbf{v}_{1}(s)\right\|_{r}^{*}\right)+C_{2} K_{W}(t) \sup _{t_{0}<s<t_{0}+t}\left(s-t_{0}\right)^{1 / 2}\|\mathbf{g}\|_{(n, \infty)} .
\end{gathered}
$$

Analogously,

$$
\begin{aligned}
|(W(t), \varphi)| & \leq C K_{\mathbf{V}}(t) \sup _{t_{0}<s<t_{0}+t}\left(s-t_{0}\right)^{n / 2\left(1 / n-1 / r^{*}\right)}\left\|w_{1}(s)\right\|_{r^{*}}\|\varphi\|_{n^{\prime}} \\
& +C K_{W}(t) \sup _{t_{0}<s<t_{0}+t}\left(s-t_{0}\right)^{n / 2\left(1 / n-1 / r^{*}\right)}\|\mathbf{v}(s)\|_{r^{*}}\|\varphi\|_{n^{\prime}},
\end{aligned}
$$

for all $\varphi \in C_{0}^{\infty}$ and all $t_{0}<t<T$. By duality,

$$
\begin{aligned}
\|W(t)\|_{n} & \leq C_{3} K_{\mathbf{V}}(t) \sup _{t_{0}<s<t_{0}+t}\left(s-t_{0}\right)^{n / 2\left(1 / n-1 / r^{*}\right)}\left\|w_{1}(s)\right\|_{r^{*}} \\
& +C_{4} K_{W}(t) \sup _{t_{0}<s<t_{0}+t}\left(s-t_{0}\right)^{n / 2\left(1 / n-1 / r^{*}\right)}\|\mathbf{v}(s)\|_{r^{*}}
\end{aligned}
$$

Let $K(t)=\max \left\{K_{\mathbf{V}}(t), K_{W}(t)\right\}$. Then we have that

$$
\begin{aligned}
\max & \left\{\|\mathbf{V}(t)\|_{n},\|W(t)\|_{n}\right\} \leq C_{*} K(t)\left\{\sup _{t_{0}<s<t_{0}+t}\left(s-t_{0}\right)^{n / 2\left(1 / n-1 / r^{*}\right)}\|\mathbf{v}(s)\|_{r^{*}}\right. \\
& +\sup _{t_{0}<s<t_{0}+t}\left(s-t_{0}\right)^{n / 2\left(1 / n-1 / r^{*}\right)}\left\|\mathbf{v}_{1}(s)\right\|_{r^{*}}+\sup _{t_{0}<s<t_{0}+t}\left(s-t_{0}\right)^{n / 2\left(1 / n-1 / r^{*}\right)}\|w(s)\|_{r^{*}} \\
& \left.+\sup _{t_{0}<s<t_{0}+t}\left(s-t_{0}\right)^{n / 2\left(1 / n-1 / r^{*}\right)}\left\|w_{1}(s)\right\|_{r^{*}}\right\}+C_{* *} K(t) \sup _{t_{0}<s<t_{0}+t}\left(s-t_{0}\right)^{1 / 2}\|\mathbf{g}\|_{(n, \infty)} .
\end{aligned}
$$

We define the constant $\widetilde{C}=C_{*}+C_{* *}$. From hypothesis of this theorem, there exists $t_{0}<t_{1} \leq T$, such that

$$
\begin{array}{r}
\sup _{t_{0}<s<t_{0}+t}\left(s-t_{0}\right)^{n / 2\left(1 / n-1 / r^{*}\right)}\left(\|w(s)\|_{r^{*}}+\left\|w_{1}(s)\right\|_{r^{*}}+\|\mathbf{v}(s)\|_{r^{*}}+\left\|\mathbf{v}_{1}(s)\right\|_{r^{*}}\right) \\
+\sup _{t_{0}<s<t_{0}+t}\left(s-t_{0}\right)^{1 / 2}\|\mathbf{g}\|_{(n, \infty)}<\frac{1}{2 \widetilde{C}}, \quad \text { for } t_{0}<t<t_{1} .
\end{array}
$$


Therefore, we have $\max \left\{\|\mathbf{V}(t)\|_{n},\|W(t)\|_{n}\right\} \leq \frac{1}{2} K(t)$, for $t_{0}<t<t_{1}$. As $K(t)$ is not increasing, we conclude that $\|\mathbf{V}(t)\|_{n} \equiv 0, \quad\|W(t)\|_{n} \equiv 0$, for $t_{0}<t \leq t_{1}$ and hence $\mathbf{v}(t)=\mathbf{v}_{1}(t), w(t)=$ $w_{1}(t)$, on the interval $\left[t_{0}, t_{1}\right]$.

Now, it remains to show that

$$
\mathbf{v}(t)=\mathbf{v}_{1}(t), \quad w(t)=w_{1}(t), \text { on }\left[t_{0}, T\right)
$$

By definition, we have that

$$
\begin{aligned}
& \sup _{t_{1}<s<T}\|\mathbf{v}(s)\|_{r^{*}}+\sup _{t_{1}<s<T}\left\|\mathbf{v}_{1}(s)\right\|_{r^{*}} \equiv M_{1}<\infty, \\
& \sup _{t_{1}<s<T}\|w(s)\|_{r^{*}}+\sup _{t_{1}<s<T}\left\|w_{1}(s)\right\|_{r^{*}} \equiv M_{2}<\infty .
\end{aligned}
$$

Thus, in order to conclude (3.68) we use the following proposition

Proposition 3.17 There exists a constant $\xi=\xi\left(n, r, M_{1}, M_{2}\right)$ such that, if $\mathbf{v}=\mathbf{v}_{1}$ and $w=w_{1}$ on $\left[t_{0}, \tau\right]$ for all $\tau \in\left[t_{1}, T\right)$, then $\mathbf{v}=\mathbf{v}_{1}$ and $w=w_{1}$ in $\left[t_{0}, \tau+\xi\right]$.

Proof. We define $D_{\mathbf{V}}(t) \equiv \sup _{\tau<s<t}\|\mathbf{V}(s)\|_{n}$ and $D_{W}(t) \equiv \sup _{\tau<s<t}\|W(s)\|_{n}$. Working similarly as the calculus in (3.66)-(3.67), we have

$$
\begin{aligned}
|(\mathbf{V}(t), \boldsymbol{\phi})| & \leq \int_{\tau}^{t}\left(\|\mathbf{v}(s)\|_{r^{*}}+\left\|\mathbf{v}_{1}(s)\right\|_{r^{*}}\right)\|\mathbf{V}(s)\|_{n}\left\|\nabla e^{-(t-s) \mathbf{A}} \boldsymbol{\phi}\right\|_{p} d s \\
& +\int_{\tau}^{t}(t-s)^{-1 / 2}\|W(s)\|_{n}\|\mathbf{g}\|_{(n, \infty)}\|\boldsymbol{\phi}\|_{n^{\prime}} \\
& \leq C M_{1} D_{\mathbf{V}}(t-\tau)^{\frac{r^{*}-n}{2 r^{*}}}\|\boldsymbol{\phi}\|_{n^{\prime}}+C D_{W}(t)(t-\tau)^{1 / 2}\|\mathbf{g}\|_{(n, \infty)}\|\boldsymbol{\phi}\|_{n^{\prime}},
\end{aligned}
$$

for all $\phi \in \mathbf{C}_{0, \sigma}^{\infty}$ and all $\tau<t<T$. Analogously, for any $\varphi \in C_{0}^{\infty}$ and any $t, \tau<t<T$, we obtain: $\|W(t)\|_{n} \leq C\left(M_{2} D_{\mathbf{V}}(t)+M_{1} D_{W}(t)\right)(t-\tau)^{\frac{r^{*}-n}{2 r^{*}}}\|\varphi\|_{n^{\prime}}$. Let $D(t) \equiv \max \left\{D_{\mathbf{V}}(t), D_{W}(t)\right\}$ and $M \equiv \max \left\{M_{1}, M_{2}\right\}$. Then we have

$$
\max \left(\|\mathbf{V}(t)\|_{n},\|W(t)\|_{n}\right) \leq \widetilde{C} M D(t)(t-\tau)^{\frac{r^{*}-n}{2 r^{*}}},
$$

for $\widetilde{C}=\max \left\{C M\|\phi\|_{n^{\prime}}, C M\|\varphi\|_{(n, \infty)}, C\|\mathbf{g}\|_{n^{\prime}} T^{n / 2 r^{*}}\|\phi\|_{n^{\prime}}\right\}$.

Let we define $\xi$ as $\xi \equiv \frac{1}{(4 \widetilde{C} M)^{\frac{2 r^{*}}{r^{*}-n}}}$. Then, $D(\tau+\xi) \leq \frac{1}{2} D(\tau+\xi)$. Consequently, $D(\tau+\xi)=0$. This implies that $\mathbf{v}=\mathbf{v}_{1}$ and $w=w_{1}$ on $\left[t_{0}, \tau+\xi\right]$.

Now we prove the existence part of Theorem 3.10. Being $(\mathbf{v}, w)$ the integral solution of (3.34)-(3.35), we can prove the time Hölder continuity of

$$
\widetilde{\mathbf{F}}(\mathbf{v}, w) \equiv-P(\mathbf{v} \cdot \nabla \mathbf{v})+P(w \mathbf{g}), \quad \widetilde{G}(\mathbf{v}, w) \equiv-(\mathbf{v} \cdot \nabla w)+f,
$$

in the $L^{r^{*}}$-space. Indeed, we follow the ideas of Kozono and Ogawa [12] (Lemma A.4), and we use the hypothesis of Hölder continuity of function $f$ (that follows using a standard argument). 
Finally, using Theorem 3.11 of [17], we conclude that the integral solution $(\mathbf{v}, w)$ verifies Definition 3.9. Hence we conclude the proof of Theorem 3.10.

Proof of Theorem 2.3. Let $(\mathbf{u}, \theta)$ be the periodic solution of $(2.8),(2.9)$ given by Theorem 2.2. As $\mathbf{u} \in Y, \theta \in X$, from Lemma 3.4 we have that $\mathbf{u} \in B C\left(\mathbb{R} ; \mathbf{L}_{\sigma}^{(n, \infty)}(\Omega) \cap \mathbf{L}^{\left(q^{*}, \infty\right)}(\Omega)\right), \theta \in$ $B C\left(\mathbb{R} ; L^{(n, \infty)}(\Omega) \cap L^{\left(q^{*}, \infty\right)}(\Omega)\right)$. Let $T$ defined by (3.33). By Theorem 3.10, for each $t_{0} \in \mathbb{R}$, there exists a unique strong solution $(\mathbf{v}, w)$ of $(1.1)-(1.5)$ on $\left(t_{0}, t_{0}+T\right)$ with the initial data $\left(\mathbf{u}\left(t_{0}\right), \theta\left(t_{0}\right)\right)$. From $(3.25),(3.32)$ we have

$$
\begin{gathered}
\sup _{t_{0}<t<t_{0}+T}\|\mathbf{v}(t)\|_{\left(q^{*}, \infty\right)}+\sup _{t_{0}<t<t_{0}+T}\|\nabla \mathbf{u}(t)\|_{(q, \infty)} \leq C_{7,1}, \\
\sup _{t_{0}<t<t_{0}+T}\|w(t)\|_{\left(q^{*}, \infty\right)}+\sup _{t_{0}<t<t_{0}+T}\|\nabla \theta(t)\|_{(q, \infty)} \leq C_{7,2},
\end{gathered}
$$

where $C_{7,1}, C_{7,2}$ are independents of $t_{0}$. Replacing $(\mathbf{a}, b)$ by $\left(\mathbf{u}\left(t_{0}\right), \theta\left(t_{0}\right)\right)$ in (3.34)-(3.35), and using estimates similar to (2.8) and (2.9) (but with integrals defined in $\left(t_{0}, t\right)$ ), we can see for $t$, $t_{0}<t<t_{0}+T$

$$
\begin{aligned}
\mathbf{u}(t)-\mathbf{v}(t) & =-\int_{t_{0}}^{t} e^{-(t-s) \mathbf{A}} P[((\mathbf{u}-\mathbf{v}) \cdot \nabla) \mathbf{u}] d s+\int_{t_{0}}^{t} e^{-(t-s) \mathbf{A}} P((\theta-w) \mathbf{g}) d s \\
& -\int_{t_{0}}^{t} e^{-(t-s) \mathbf{A}} P[(\mathbf{v} \cdot \nabla)(\mathbf{u}-\mathbf{v})](s) d s \equiv I_{1}(t)+I_{2}(t)+I_{3}(t), \\
\theta(t)-w(t) & =-\int_{t_{0}}^{t} e^{-(t-s) B}((\mathbf{u}-\mathbf{v}) \cdot \nabla \theta) d s-\int_{t_{0}}^{t} e^{-(t-s) B}(\mathbf{v} \cdot \nabla(\theta-w)) d s \\
& \equiv I_{4}(t)+I_{5}(t) .
\end{aligned}
$$

Note that, for every $t, t_{0}<t<t_{0}+T$,

$$
\left\|I_{1}(t)\right\|_{(n, \infty)} \leq C \sup _{s \in \mathbb{R}}\|\nabla \mathbf{u}(s)\|_{(q, \infty)} \sup _{t_{0}<s<t_{0}+t}\|(\mathbf{u}-\mathbf{v})(s)\|_{(n, \infty)}\left(t-t_{0}\right)^{(1-n / 2 q)},
$$

where $C=C(n, q)$ is independent of $t_{0}$. Moreover, for any $\phi \in \mathbf{C}_{0, \sigma}^{\infty}$ and $t, t_{0}<t<t_{0}+T$ :

$$
\left|\left(I_{2}(t), \phi\right)\right| \leq C \sup _{t_{0}<s<t_{0}+t}\|\mathbf{v}(s)\|_{\left(q^{*}, \infty\right)} \sup _{t_{0}<s<t_{0}+t}\|\mathbf{u}(s)-\mathbf{v}(s)\|_{(n, \infty)}\left(t-t_{0}\right)^{1-n / 2 q}\|\boldsymbol{\phi}\|_{\left(n^{\prime}, 1\right)},
$$

being $C$ independent of $t_{0}$. By duality, we have that for any $t, t_{0}<t<t_{0}+T$,

$$
\begin{aligned}
\left\|I_{2}(t)\right\|_{(n, \infty)} \leq C \sup _{t_{0}<s<t_{0}+t} & \|\mathbf{v}(s)\|_{\left(q^{*}, \infty\right)} \sup _{t_{0}<s<t_{0}+t}\|\mathbf{u}(s)-\mathbf{v}(s)\|_{(n, \infty)}\left(t-t_{0}\right)^{1-n / 2 q}, \\
\left\|I_{3}(t)\right\|_{(n, \infty)} & \leq C \int_{t_{0}}^{t}(t-s)^{-n / 2 b}\|\theta(s)-w(s)\|_{(n, \infty)}\|\mathbf{g}\|_{(b, \infty)} \\
& \leq C \sup _{t_{0}<s<t_{0}+t}\|\theta(s)-w(s)\|_{(n, \infty)}\left(t-t_{0}\right)^{1-n / 2 b},
\end{aligned}
$$

with $C$ independent of $t_{0}$. Analogously we have that for any $t \in\left(t_{0}, t_{0}+T\right)$,

$$
\begin{gathered}
\left\|I_{4}(t)\right\|_{(n, \infty)} \leq C \sup _{t_{0}<s<t_{0}+t}\|\nabla \theta(s)\|_{(\tilde{q}, \infty)} \sup _{t_{0}<s<t_{0}+t}\|(\mathbf{u}-\mathbf{v})(s)\|_{(n, \infty)}\left(t-t_{0}\right)^{(1-n / 2 \tilde{q})}, \\
\left\|I_{5}(t)\right\|_{(n, \infty)} \leq C \sup _{t_{0}<s<t_{0}+t}\|\mathbf{v}(s)\|_{\left(q^{*}, \infty\right)} \sup _{t_{0}<s<t_{0}+t}\|\theta(s)-w(s)\|_{(n, \infty)}\left(t-t_{0}\right)^{1-n / 2 q},
\end{gathered}
$$


with $C$ independent of $t_{0}$. From (3.69)-(3.77) follow that for all $t \in\left(t_{0}, t_{0}+T\right)$,

$$
\begin{aligned}
\|\mathbf{u}(t)-\mathbf{v}(t)\|_{(n, \infty)} & \leq C_{1}\left(\sup _{t_{0}<s<t_{0}+t}\|\mathbf{u}(s)-\mathbf{v}(s)\|_{(n, \infty)}\left(t-t_{0}\right)^{1-n / 2 q}\right. \\
& \left.\left.+\sup _{t_{0}<s<t_{0}+t}\|\theta(s)-w(s)\|_{(n, \infty)}\right)\left(t-t_{0}\right)^{1-n / 2 b}\right), \\
\|\theta(t)-w(t)\|_{(n, \infty)} & \leq C_{2}\left(\sup _{t_{0}<s<t_{0}+t}\|\mathbf{u}(s)-\mathbf{v}(s)\|_{(n, \infty)}\left(t-t_{0}\right)^{1-n / 2 \widetilde{q}}\right. \\
& \left.+\sup _{t_{0}<s<t_{0}+t}\|\theta(s)-w(s)\|_{(n, \infty)}\left(t-t_{0}\right)^{1-n / 2 q}\right),
\end{aligned}
$$

where $C_{1}, C_{2}$ are independents of $t_{0}$. Taking $E(t)=\max \left\{\|\mathbf{u}(t)-\mathbf{v}(t)\|_{(n, \infty)},\|\theta(t)-w(t)\|_{(n, \infty)}\right\}$, for all $t \in\left(t_{0}, T_{0}+T\right)$, from (3.78)-(3.79) it follows that for all $t_{0}<t<t_{0}+T$

$$
E(t) \leq C_{3} \sup _{t_{0}<s<t_{0}+t} E(s)\left(t-t_{0}\right)^{1-n / 2 p},
$$

where $p=\max (b, q)$. Therefore, $E(t) \leq C_{3} \sup _{t_{0}<s<t_{0}+t} E(s) T^{1-n / 2 p}$.

Taking $\varsigma \equiv \min \left\{\left(1 / 2 C_{3}\right)^{2 p /(2 p-n)}, T\right\}$, we conclude that: for every $t, t_{0}<t<t_{0}+T$,

$$
E(t) \leq C_{3} \varsigma^{1-n / 2 p} \sup _{t_{0}<s<t_{0}+t} E(T) \leq 1 / 2 \sup _{t_{0}<t<t_{0}+t} E(s),
$$

and hence we obtain $E(t) \equiv 0$ on $\left[t_{0}, t_{0}+\varsigma\right)$. Since $\varsigma$ can be taken independently of $t_{0}$, we have $E(t) \equiv 0$ on $\left[t_{0}, t_{0}+T\right)$. This implies that $\mathbf{u}=\mathbf{v}$ on $\left[t_{0}, t_{0}+T\right)$ and $\theta=w$ on $\left[t_{0}, t_{0}+T\right)$. Finally, as $t_{0}$ is an arbitrary time in Theorem 3.10, we conclude that $(\mathbf{u}, \theta)$ is the required solution in Theorem 2.3.

\section{References}

[1] Bergh, J., Löfström, J.: Interpolation Spaces, Springer-Verlag, Berlin, 1976

[2] Borchers, W., Miyakawa, T.: On stability of exterior stationary Navier-Stokes flows. Acta Math. 174, 311-382 (1995)

[3] Cannon, J.R., DiBenedetto, E.: The initial value problem for the Boussinesq equations with data in $L^{p}$, in Approximation Methods for Navier-Stokes Problems, Edited by Rautmann, R., Lect. Notes in Math., Springer-Verlag, Berlin, 771, 1980

[4] Chandrasekhar, S.: Hidrodinamic and Hydromagnetic Stability, New york, 1981

[5] Ferreira, L.C.F., Villamizar-Roa, E.J.: Well-posedness and asymptotic behaviour for the convection problem in $\mathbb{R}^{n}$. Nonlinearity, 19, 2169-2191 (2006)

[6] Ferreira, L.C.F., Villamizar-Roa, E.J.: Existence of solutions to the convection problem in a pseudomeasure-type space. Proc. R. Soc. Lond. Ser. A Math. Phys. Eng. Sci. 464, no. 2096, 1983-1999 (2008) 
[7] Fife, P.C., Joseph, D.D.: Existence of convective solutions of the generalized Bérnard problem which are analytic in their norm. Arch. Rational Mech. Anal. 33, 116-138 (1969)

[8] Fujiwara, D., Morimoto, H.: An $L^{r}$-Theorem of the Helmholtz decomposition of vectos fields. Fac.Sci.Univ.Tokio, sec. IA. 24, 658-700 (1977)

[9] Giga, Y.: Analiticy of the semigroup generated by the Stokes operator in $L_{r}$ spaces. Math. Z. 187, 297-329 (1981)

[10] Kato, T.: Strong $L^{p}$-solutions of the Navier-Stokes equations in $\mathbf{R}^{m}$, with applications to weak solutions. Math. Z. 187, 471-480 (1984)

[11] Kozono, H., Nakao, M.: Periodic solutions of the Navier-Stokes equations in unbounded domains. J. Tohoku Math. 48, 33-50 (1996)

[12] Kozono, H., Ogawa, T.: Some $L^{p}$ estimate for the exterior Stokes flow and an application to the nonstationary Navier-Stokes equations. Indiana Univ. Math. J. 41, 789-808 (1992)

[13] Lucaszewicz, G., Ortega-Torres, E.E., Rojas-Medar, M.A.: Strong periodic solutions for a class of abstract evolution equations. Nonlinear Analysis. 54, 1045-1056 (2003)

[14] Maremonti, P.: Existence and stability of time periodic solutions of the Navier-Stokes equations in whole space. Nonlinearity. 4, 503-529 (1991)

[15] Maremonti, P.: Some theorems of existence for solutions of the Navier-Stokes equations with slip boundary conditions in half-space. Rich. Mat. 40, 81-135 (1991)

[16] O’Neil, R.: Convolution operators and $L(p, q)$ spaces. Duke Math. J. 30, 129-142 (1963)

[17] Tanabe, H.: Equations of evolution, London, Pitman, 1979

[18] Taniuchi, Y.: On stability of periodic solutions of the Navier-Stokes equations in unbounded domains. Hokkaido Math. J. 28, 147-173 (1999)

[19] Yamasaki, M.: Solutions in Morrey Spaces of the Navier-Stokes Equations with TimeDependent External Force. Funk. Ekva. 43, 419-460 (2000) 